\title{
The AvrB_AvrC Domain of AvrXccC of Xanthomonas campestris pv. campestris Is Required to Elicit Plant Defense Responses and Manipulate ABA Homeostasis
}

\author{
Yi-Ping Ho, ${ }^{1}$ Choon Meng Tan, ${ }^{1}$ Meng-Ying Li, ${ }^{1}$ Hong Lin, ${ }^{1}$ Wen-Ling Deng, ${ }^{2}$ and Jun-Yi Yang ${ }^{1,3,4}$ \\ ${ }^{1}$ Institute of Biochemistry, National ChungHsing University, Taichung 40227, Taiwan; ${ }^{2}$ Department of Plant Pathology, \\ National ChungHsing University, Taichung 40227, Taiwan; ${ }^{3}$ Institute of Biotechnology, National ChungHsing University, \\ Taichung 40227, Taiwan; ${ }^{4}$ Agricultural Biotechnology Center, National ChungHsing University, Taichung 40227, Taiwan
}

Submitted 30 June 2012. Accepted 9 November 2012.

Plant disease induced by Xanthomonas campestris pv. campestris depends on type III effectors but the molecular basis is poorly understood. Here, AvrXecC $_{8004}$ was characterized, and it was found that the AvrB_AvrC domain was essential and sufficient to elicit defense responses in an Arabidopsisresistant ecotype (Col-0). An upregulation of genes in responding to the AvrB_AvrC domain of AvrXccC ${ }_{8004}$ was shown in a profile of host gene expression. The molecular changes were correlated with morphological changes observed in phenotypic and ultrastructural characterizations. Interestingly, the abscisic acid (ABA)-signaling pathway was also a prominent target for the AvrB_AvrC domain of AvrXccC ${ }_{8004}$. The highly elicited $N C E D 5$, encoding a key enzyme of ABA biosynthesis, was increased in parallel with ABA levels in AvrXcc $_{8004}$ transgenic plants. Consistently, the $X$. campestris pv. campestris 8004 AavrXccC mutant was severely impaired in the ability to manipulate the accumulation of $\mathrm{ABA}$ and induction of $\mathrm{ABA}$-related genes in challenged leaves. Moreover, exogenous application of ABA also enhanced the susceptibility of Arabidopsis to the $X$. campestris pv. campestris strains. These results indicate that the AvrB_AvrC domain of $\mathrm{AvrXccC}_{\mathbf{8 0 0 4}}$ alone has the activity to manipulate ABA homeostasis, which plays an important role in regulating the interactions of $X$. campestris pv. campestris and Arabidopsis.

Xanthomonas campestris pv. campestris is a gram-negative bacterium that is responsible for the black rot disease, one of the most destructive diseases on cruciferous plants (Williams 1980). This plant pathogen infects numerous important species of crucifers, including broccoli, mustard, cabbage, cauliflower, radish, and turnip, as well as the model plant Arabidopsis thaliana (Simpson and Johnson 1990). The successful infection requires protein secretion systems to deliver virulence factors into intracellular space or translocate directly into host cells (Büttner and Bonas 2010). One of the important secretion systems is the type III secretion system, which injects effector

Corresponding author: J.-Y. Yang; E-mail: jyang@nchu.edu.tw

* The $\boldsymbol{e}$-Xtra logo stands for "electronic extra" and indicates that six supplementary tables and four supplementary figures are published online.

C 2013 The American Phytopathological Society proteins into host cell to interfere with host immunity response and plant cellular process to ensure bacterial multiplication and disease progression (White et al. 2009). In resistant plants, the virulence effectors might be recognized indirectly by host resistance $(\mathrm{R})$ proteins through the virulence activities of effectors (DeYoung and Innes 2006). These recognition events often trigger the hypersensitive response, resulting in rapid localized cell death that restricts pathogen growth (Coll et al. 2011).

The outcomes of the plant-pathogen interactions depend on the balance between plant defense and pathogen counterdefense. The plant immune system contains two levels of defense responses: pathogen-associated molecular pattern (PAMP)-triggered immunity and effector-triggered immunity. PAMP-triggered immunity is a primary immune response activated by direct recognition of PAMPs, and effector-triggered immunity is a rapid immune response activated by indirect recognition of pathogen effectors (Dodds and Rathjen 2010). The signaling events induced by PAMPs or effectors are mainly regulated by plant hormones which activate successful defense responses against pathogen infections (Pieterse et al. 2009; Verhage et al. 2010). Three phytohormones, including salicylic acid (SA), jasmonic acid (JA), and ethylene, are known as primary signal molecules in regulating plant immune responses. Recently, other phytohormones, including abscisic acid (ABA), brassinosteroid, gibberellins, cytokinin, and auxin, also emerged as important components involved in plant immune responses (Bari and Jones 2009; Robert-Seilaniantz et al. 2007; Spoel and Dong 2008).

Pathogens have evolved various ways to interfere with plant immune responses. One of the striking ways is to produce plant hormones by pathogens themselves, such as Agrobacterium tumefaciens. A. tumefaciens is a crown gall disease bacterium that produces auxin to suppress host defense responses, thus enhancing host susceptibility to diseases (Dunoyer et al. 2006; Navarro et al. 2006). In addition, coronatine, a mimic molecule of JA, is synthesized by Pseudomonas syringae to affect JA homeostasis that antagonizes SA-mediated defense responses (Cui et al. 2005; Schulze-Lefert and Robatzek 2006). Another strategy is to modulate plant hormone signaling by type III effectors such as $P$. syringae AvrPtoB, which appears to be able to elevate ABA levels to overcome plant basal defense (de Torres-Zabala et al. 2007). Moreover, P. syringae AvrB appears to regulate JA signaling, which interferes with SA response and promotes plant susceptibility (Shang et al. 2006). 
Functional characterization of type III effectors is one of the key elements to understanding the molecular mechanisms of plant-pathogen interactions. In fact, the big challenge in systematic characterization of type III effectors is functional redundancy, in which mutations of individual effector genes usually do not affect the bacterial virulence or only have a subtle effect (Castañeda et al. 2005). In the past, different experimental approaches have been applied to study the mode of actions of individual effectors, which provided novel insights into the plantpathogen interactions (Munkvold and Martin 2009; Salomon et al. 2011; Wilton and Desveaux 2010). Now, although more than 23 type III effectors were reported in X. campestris pv. campestris 8004 based on bioinformatic predications and experimental analyses, the molecular functions in pathogenesis of most effectors remain to be investigated (Büttner and Bonas 2010; Canonne et al. 2011; Jiang et al. 2008; Jiang et al. 2009; Wang et al. 2007; White et al. 2009; Xu et al. 2008).

Previous studies have shown that $X$. campestris pv. campestris type III effector $\mathrm{AvrXccC}_{8004}$ functions as an avirulence factor which is able to induce effector-triggered innate immunity and is responsible for disease resistance in Arabidopsis Col-0 (Rong et al. 2010; Wang et al. 2007). The resistance required NDR1,
$R A R 1$, and $S G T 1 b$ to defend against $X$. campestris pv. campestris 8004 ; however, the responsible $\mathrm{R}$ protein for recognition of $\mathrm{AvrXccC}_{8004}$ has not been identified. $\mathrm{AvrXccC}_{8004}$ not only functions as an avirulence factor but also acts as a virulence factor. In Arabidopsis rarl and sgtlb mutants, X. campestris pv. campestris 8004 -inoculated leaves showed higher pathogenicity responses than those inoculated with $\triangle a v r X c c C$ strain (Rong et al. 2010). AvrXccC $\mathrm{C}_{8004}$ contains a conserved AvrB_AvrC domain between 113 to 438 amino acids (aa) which shared $61 \%$ similarity with the avirulence effector AvrB from $P$. syringae pv. glycinea (Gopalan et al. 1996; Wang et al. 2007). In this study, we used the inducible expression system to analyze the activities of AvrXccC $\mathrm{C}_{8004}$ in Arabidopsis transgenic plants and focused on the functional studies of the AvrB_AvrC domain, which has not been characterized.

\section{RESULTS}

AvrB_AvrC domain of AvrXecC ${ }_{8004}$ is essential and sufficient to elicit defense responses in Arabidopsis.

Arabidopsis transgenic plants carried full-length AvrXccC $\mathrm{C}_{8004}$ under the control of a $\beta$-estradiol-inducible promoter were
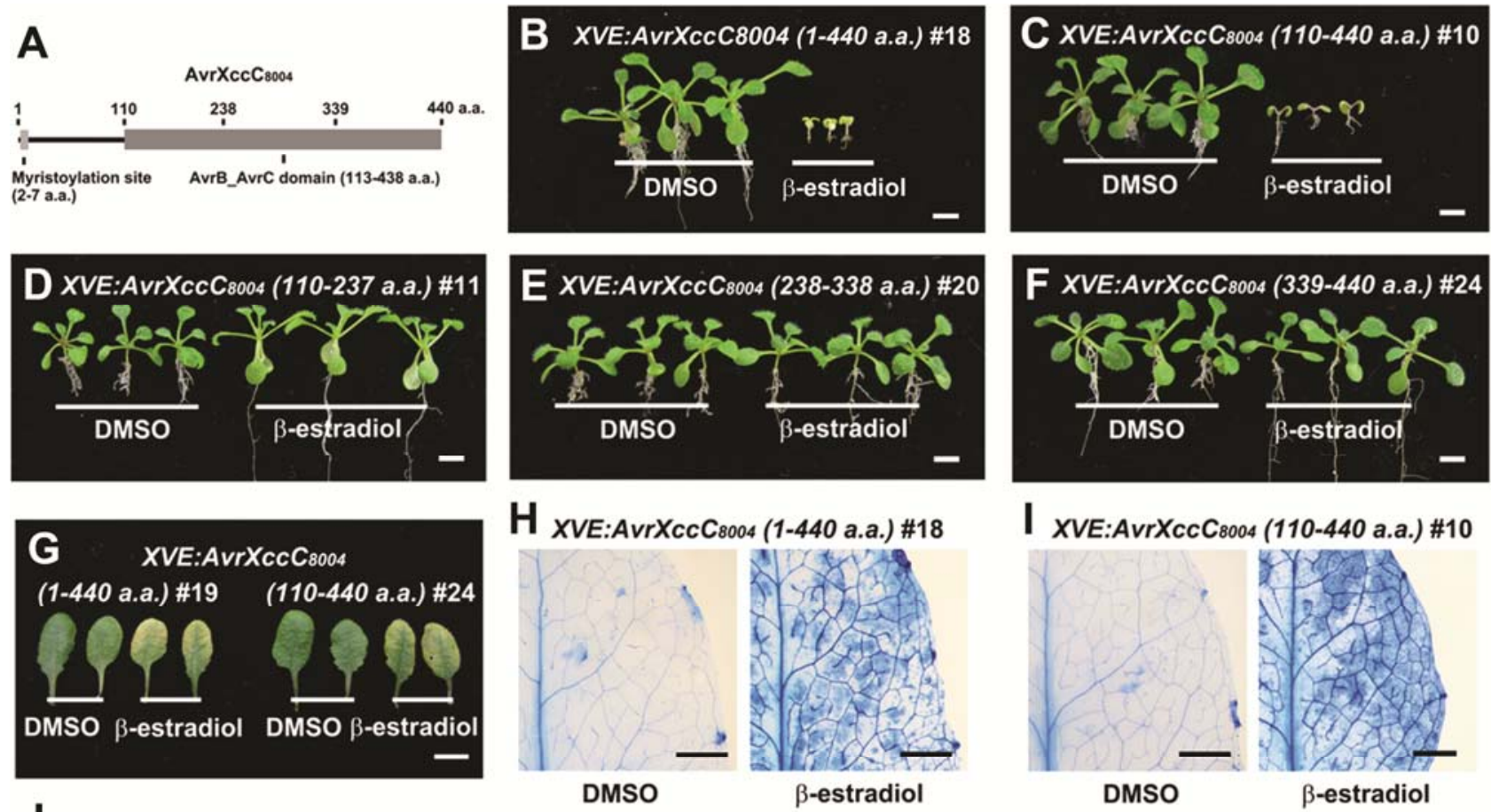

J

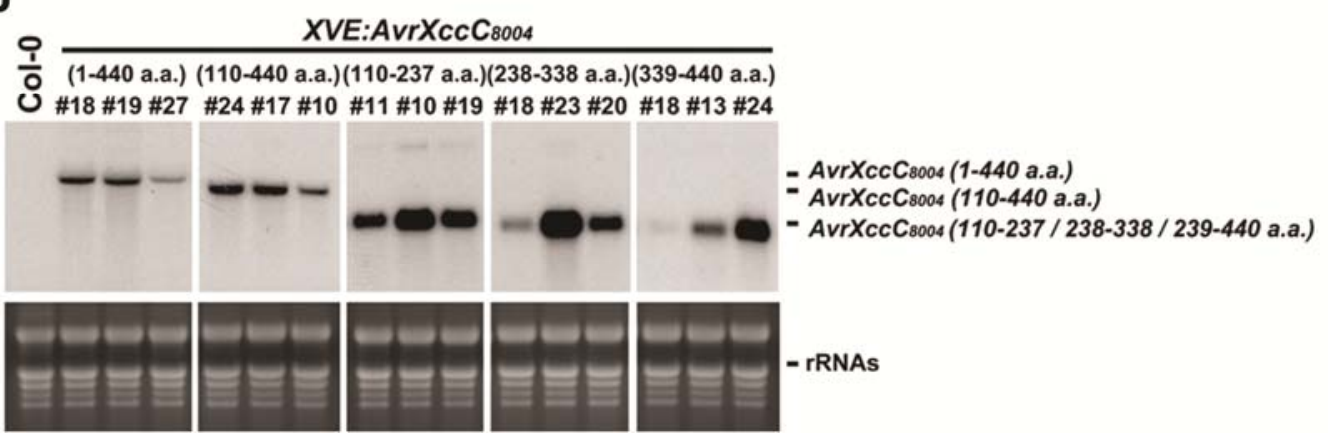

Fig. 1. Expression of $\mathrm{AvrXccC}_{8004}$ (110 to 440 amino acids [aa]) was sufficient to elicit cell death in Arabidopsis. A, Schematic representation of AvrXccC $_{8004}$. The light-gray and dark-gray color boxes present the myristoylation site and AvrB_AvrC domain of AvrXccC 8004 , respectively. B through $\mathbf{F}$, Phenotypes of 4-week-old Arabidopsis transgenic plants carried full length or a deletion series of $A v r X c c C_{8004}$ driven by XVE promoter. Scale bars: 5 mm. G, Leaf phenotypes of $X V E: A v r X c c C_{8004}(1$ to $440 \mathrm{aa})$ and $X V E: A v r X c c C_{8004}(110$ to 440 aa) transgenic plants. Scale bar: 5 mm. H and I. Trypan blue staining of transgenic plants expressing $\operatorname{AvrXccC}_{8004}$ (1 to 440 aa) and $\operatorname{AvrXccC}_{8004}$ (110 to 440 aa). Scale bars: 1 mm. J, Transcript levels of a deletion series of $A v r X c c C_{8004}$ in $\beta$-estradiol-treated transgenic plants. Stained rRNAs bands were used as a loading control. 
generated (Fig. 1A). Compared with a dimethyl sulfoxide (DMSO) control, transgenic plants expressing AvrXccC $\mathrm{C}_{8004}$ failed to grow after germination and developed chlorotic cotyledons upon $20 \mu \mathrm{M} \beta$-estradiol treatment (Fig. 1B). Arrested growth associated with chlorotic cotyledons in $X V E: A v r X c c C_{8004}$ ( 1 to 440 aa) transgenic plants may be accounted for the actions of AvrB_AvrC domain. To test this hypothesis, we introduced $X V E: A v r X c c C_{8004}(110$ to 440 aa) into Arabidopsis and analyzed the phenotypes in the presence of $\beta$-estradiol. Similar to $X V E: A v r X c c C_{8004}$ (1 to 440 aa) transgenic plants, the expression of $\mathrm{AvrXccC}_{8004}(110$ to $440 \mathrm{aa})$ resulted in arrested growth of seedlings and chlorosis in cotyledons (Fig. 1C). To define the subdomain responsible for developmental defects in Arabidopsis, AvrB_AvrC domain of AvrXcc $\mathrm{C}_{8004}$ was divided into three parts: 110 to 237,238 to 338 , and 339 to 440 aa (Fig. 1A). Transgenic plants carrying $X V E: A v r X c c C_{8004}$ (110 to 237aa), $X V E: A v r X c c C_{8004}$ (238 to 338 aa), or $X V E: A v r X c c C_{8004}$ (339 to 440 aa) were generated and analyzed in the presence of $\beta$-estradiol. Compared with the DMSO control, no developmental defects were found in individual transgenic lines (Fig. $1 \mathrm{D}$ to $\mathrm{F}$ ). Further examination of the expression levels of $A v r X c c C_{8004}$ (110 to 237 aa), $A v r X c c C_{8004}$ (238 to 338 aa), and $A v r X c c C_{8004}$ (339 to 440 aa) illustrated that all transcripts were accumulated at levels even higher than those of $A v r X c c C_{8004}$ (1 to 440 aa) and $A v r X c c C_{8004}$ (110 to 440 aa) upon $\beta$-estradiol treatment (Fig. 1J). These results indicate that the intact AvrB_AvrC domain of $A v r X c c C_{8004}$ was essential to cause the developmental defects in Arabidopsis.

To further confirm whether the developmental defects shown in transgenic plants were due to the incompatible interactions between Arabidopsis and the AvrB_AvrC domain of $\mathrm{AvrXccC}_{8004}, \quad X V E: A v r X c c C_{8004} \quad(1$ to 440 aa) and $X V E: A v r X c c C_{8004}$ (110 to 440 aa) transgenic plants were grown in soil and sprayed with $20 \mu \mathrm{M} \beta$-estradiol after plants were 4 weeks old. Compared with the DMSO control, chlorotic phenotypes were observed in the leaves of transgenic plants 2 days after spraying (Fig. 1G). Further examination using trypan blue staining confirmed that $\mathrm{AvrXccC}_{8004}(110$ to 440 aa) could elicit cell death as well as full-length $\mathrm{AvrXccC}_{8004}$ (Fig. 1H and I). By contrast, no cell deaths were found in transgenic plants expressing $\mathrm{AvrXccC}_{8004}$ (110 to 237aa), $\mathrm{AvrXccC}_{8004}$ (238 to 338 aa), or $\mathrm{AvrXccC}_{8004}$ (339 to 440 aa) (Supplementary Fig. S1). These results suggest that the AvrB_AvrC domain of $\mathrm{AvrXccC}_{8004}$ is sufficient to elicit the incompatible interactions which result in cell death phenotype.

\section{Ultrastructural characterizations of transgenic plants expressing AvrB_AvrC domain of AvrXcc 8004 $_{\text {. }}$}

To understand the Arabidopsis cellular responses to the actions of the AvrB_AvrC domain of AvrX $\mathrm{XcC}_{8004}$ in detail, leaf sections of $X V E: A v r X c c C_{8004}(110$ to 440 aa) transgenic plants were examined. Under light microscopy, differences were observed in cell integrity of $X V E: A v r X c c C_{8004}$ (110 to 440 aa) transgenic plants between DMSO and $\beta$-estradiol treatments (Fig. 2A and E). For DMSO treatment, cells were arranged with a proper space (Fig. 2A). The roughly pillshaped chloroplasts, which were tightly connected with each other and pushed against the cell periphery, were frequently observed (Fig. 2A). By contrast, after $\beta$-estradiol treatment, cells were separated by a widened intercellular space, and the chloroplasts were not only flat and fewer but also distant from the cell periphery (Fig. 2E). These phenomena can indicate the loss of turgor pressure in vacuoles caused by plasmolysis (Hatsugai et al. 2009). We further examined the exchanges of subcellular ultrastructure using transmission electron microscopy (TEM) upon $\beta$-estradiol treatment (Fig. 2F to I). Compared with DMSO control (Fig. 2B and C), the
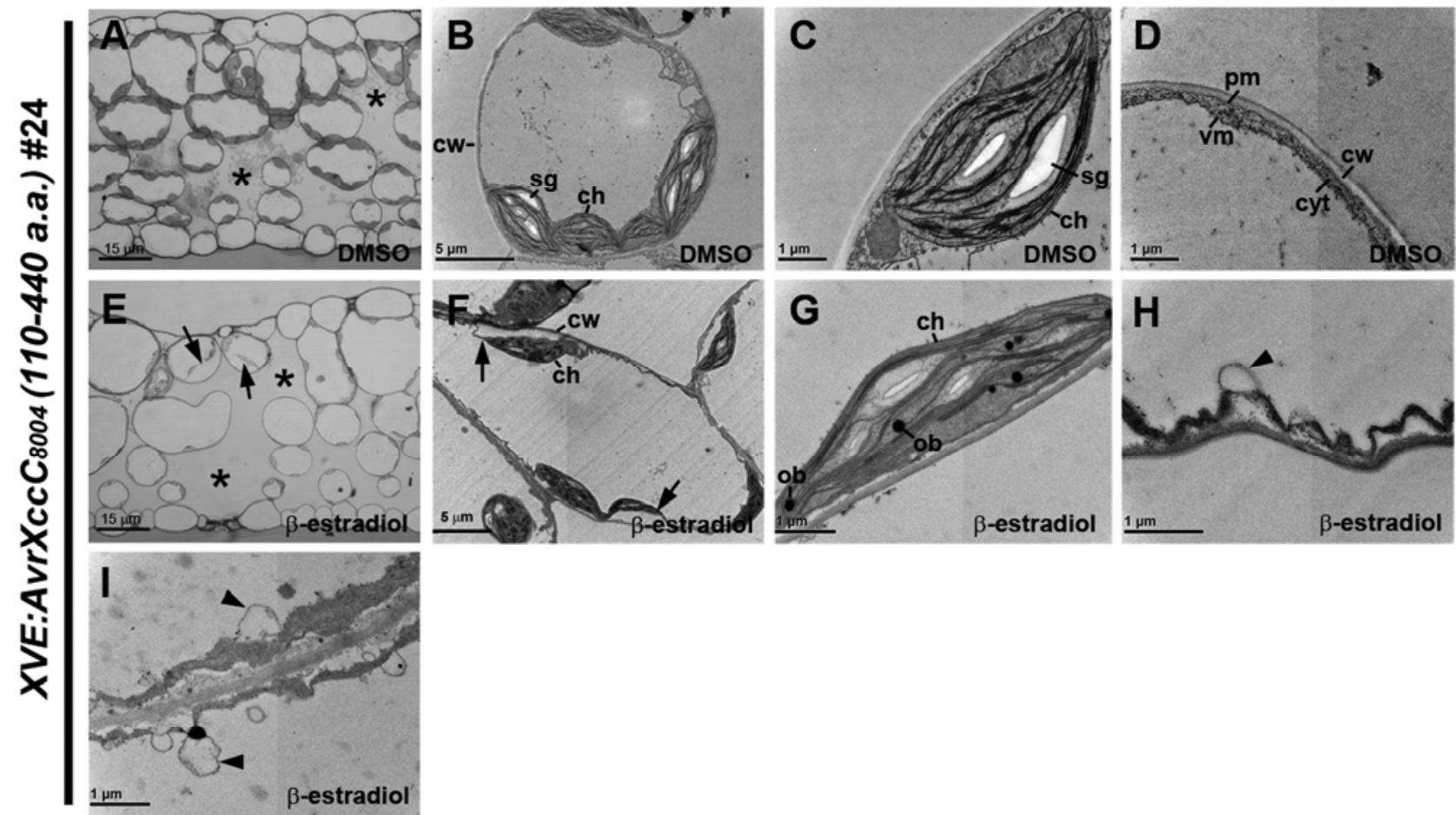

Fig. 2. Cellular responses in Arabidopsis transgenic plants expressing AvrXccC ${ }_{8004}$ (110 to 440 amino acids). Rosette leaves from 5-week-old Arabidopsis transgenic plants were collected for embedding with resin. A and E, Semi-thin sections $(1 \mu \mathrm{m})$ were stained with toluidine blue for general view of morphological changes by light microscopy. B through $\mathbf{D}$ and $\mathbf{F}$ through $\mathbf{I}$, For ultrastructural characterizations, ultrathin sections ( $80 \mathrm{~nm}$ ) were processed and viewed by transmission electron microscopy. Images were collected and compared between $\beta$-estradiol treatment and dimethyl sulfoxide (DMSO) control. Visible organelles were indicated as follows: cw, cell wall; cyt, cytosol; pm, plasma membrane; vm, vacuolar membrane; ch, chloroplast; ob, oil body; sg, starch granule; asterisks, intercellular spaces; arrows, detached chloroplasts; arrowheads, vesicles. 
reduced number of starch granules and presence of oil droplets (plastoglobuli) were frequently observed (Fig. 2F and $\mathrm{G})$, which were usually accompanied by cell death under stress conditions (Mizuno et al. 2010; Wright et al. 2009). Moreover, plasma membranes became rippled and associated with small vesicles (Fig. $2 \mathrm{H}$ and I) compared with the DMSO control (Fig. 2D). Consistent with the morphological changes shown in Figure 1C and I, the ultrastructural characterizations confirm that the AvrB_AvrC domain of AvrXccC $\mathrm{C}_{8004}$ is important for the incompatible interactions in Arabidopsis.

In addition, leaf sections of $X V E: A v r X c c C_{8004}(1$ to 440 aa) transgenic plants were also examined. Similar to the ultrastructural changes observed in $X V E: A v r X c c C_{8004}(110$ to 440 aa) transgenic plants, the widened intercellular space, detached chloroplasts with slim shape, and presence of oil droplets together with twisted plasma membranes and small vesicles were also observed in $X V E: A v r X c c C_{8004}$ (1 to 440 aa) transgenic plants after $\beta$-estradiol treatment (Supplementary Fig. S2A through I).

\section{RNA-Seq profiling analysis using high-throughput next-generation sequencing.}

To correlate the phenotypic alterations with molecular changes, transcriptome data of $X V E: A v r X c c C_{8004}$ (110 to 440 aa) transgenic plants upon DMSO and $\beta$-estradiol treatments were analyzed. After quality trim, 24.9 million reads and 24.3 billion bases were generated by Illumina 100-bp single-end sequencing from two cDNA libraries (Supplementary Table $\mathrm{S} 1$ ). Among them, $89 \%$ of the sequenced reads from DMSO treatment were mapped to 20,845 Arabidopsis genes and $86 \%$ of the sequenced reads from $\beta$-estradiol treatment were mapped to 21,102 Arabidopsis genes (Supplementary Table S2). The expression levels of mapped genes from two samples were quantified and converted to PRKM values based on sequenced reads. Stable housekeeping genes (Gutierrez et al. 2008), including $T U B 9, A C T 7, A C T 8, U B Q 5$, and $E F 1 \alpha$, were used as reference genes. The change of reads per kilobase of exon model per million mapped reads (RPKM) values for the reference genes ranged from 0.91 -fold (ACT8) to 1.11-fold (ACT7), indicating that the expression levels of reference genes were similar across different treatments (Supplementary Table S3). These results suggest that the RNA-Seq data is reliable and can be used for further analysis.

To identify differentially expressed genes in $X V E: A v r X c c C_{8004}$ (110 to 440 aa) transgenic plants upon $\beta$-estradiol treatment, differential expression analysis using the DESeq method (Anders and Huber 2010) was performed. Compared with DMSO treatment, 771 genes with $P<0.05$ were identified after $\beta$-estradiol treatment. Among them, 693 genes were upregulated and 78 genes were downregulated. To narrow down the differentially expressed genes, a criteria with $P<0.01$ and $\log _{2}$ (fold change) $>3.0$ or $<-3.0$ was selected. As a result, 337 genes were identified. Among them, 324 genes were upregulated and 13 genes were downregulated (Supplementary Table S4). Using Gene Ontology (GO) analysis to classify the 324 differentially expressed genes revealed that several terms, including cellular process, response to stimulus, metabolic process, cell, catalytic activity, and binding, were highlighted in individual categories (Supplementary Fig. S3). In all, 98 of 337 differentially expressed genes were mapped to the "response to stimulus" term (Supplementary Table S5), which suggested that Arabidopsis could respond to the activities of AvrXccC $\mathrm{C}_{8004}$ (110 to $440 \mathrm{aa}$ ) and regulated gene expression for cellular or behavioral stimulations. Under the response to stimulus term, 38 genes were further mapped to the "defense response" term and 31 genes were further mapped to the "response to other organism" term, which indicated that the induction of
AvrXccC $_{8004}$ (110 to 440 aa) strikingly triggered the plant immune system related to pathogen infection.

\section{Multiple plant defense systems were triggered by $\mathrm{AvrXccC}_{8004}(110$ to 440 aa).}

Functional annotation on the 337 differentially expressed genes $(P<0.01)$ revealed that multiple plant defense systems were triggered in $X V E: A v r X c c C_{8004}(110$ to 440 aa) transgenic plants upon $\beta$-estradiol treatment. Remarkably, an upregulation of genes involved in the SA-mediated defense signaling network ( $\mathrm{Lu} 2009$ ) was shown in responding to $\mathrm{AvrXccC}_{8004}$ (110 to 440 aa). The transcriptome profiling data showed that pathogenesis-related (PR)1 and PR2, which are standard markers for SA-mediated defense, were induced 1,182- and 100-fold, respectively (Table 1). At the same time, transcript levels of genes involved in SA biosynthesis (EDS5, SID2, SARD1, and CBP60g) (Nawrath et al. 2002; Wang et al. 2011b; Wildermuth et al. 2001) as well as SA accumulation and signaling ( $A L D 1$, WIN3, PAD4, FMO1, NUDX6, and PDLP5) (Ishikawa et al. 2010; Lee et al. 2011; Mishina and Zeier 2006; Song et al. 2004; Wang et al. 2011a; Zhou et al. 1998) were highly elevated (Table 1). Among these genes, SID2, which encodes an isochorismate synthase that produces an immediate precursor of SA, was induced 26-fold; and PAD4, which encodes a lipaselike protein that interacts directly with EDS1 (Feys et al. 2001), an essential element for hypersensitive response, was induced 31-fold. For downstream signaling, WRKY transcription factors (WRKY51, WRKY72, WRKY8, WRKY 38, and WRKY50), which were classified into the defense response term, had higher expression levels (Table 1). In addition, other plant defense response-associated genes, including DOX1, AIG1, CML43, PARG2, UGT85A1, TRX5, CDA1, SDR3, and LOX1 (Adams-Phillips et al. 2010; Carviel et al. 2009; Chiasson et al. 2005; Hwang et al. 2011; Laloi et al. 2004; Reuber and Ausubel 1996; Vicente et al. 2012) were also highly expressed (Table 1). Among them, DOX1 and LOX1, which encode key enzymes for oxylipins that play important roles in plant defense, were induced 1,536-fold and 15-fold, respectively.

Here, in an Arabidopsis resistant ecotype (Col-0), a group of genes which encode Toll-interleukin-1 receptor (TIR) domaincontaining proteins, including TIR adaptor proteins (At5g38344, At4g19925, At1g57630, and At2g20142) and TIR nucleotide-binding site leucine-rich repeat (NBS-LRR) proteins (At5g38350, At4g11340, At5g18350, and At3g04220), were highly upregulated by $\operatorname{AvrXccC}_{8004}$ (110 to 440 aa) (Table 2). However, several genes, including $B O N 1, B O N 3, B A P 1$, $B A P 2$, and $K T I 1$ (Li et al. 2008; Li et al. 2009; Yang et al. 2006a; Yang et al. 2006b; Yang et al. 2007), which act as negative regulators to repress R-mediated defense responses, were also highly elevated by $\mathrm{AvrXccC}_{8004}(110$ to 440 aa) (Table 2 ). Thus, $\operatorname{AvrXccC}_{8004}$ (110 to 440 aa) may be able to manipulate $R$-gene-mediated responses through its virulence activities to counteract plant defense responses.

\section{Expression of AvrXccC $_{8004}(110$ to 440 aa) interfered with the ABA-signaling responses.}

In addition to defense response mediated by the SA-signaling pathway, the ABA-signaling pathway was also found to be a prominent target for $\mathrm{AvrXccC}_{8004}$ (110 to 440 aa) (Table 3). The upregulated genes were involved in ABA biosynthesis, accumulation, and signaling transduction. For ABA biosynthesis, NCED5, which encodes a 9-cis-epoxycarotenoid dioxygenase that cleavages the precursor of ABA (Frey et al. 2012), was induced 151-fold. For ABA accumulation and signaling, PDR12, which encodes an ABC transporter that mediates cellular uptake of ABA (Kang et al. 2010), was induced 85-fold; SAG113, which encodes a PP2C-type phosphatase that acts as a regulator 
of the ABA signaling pathway (Zhang et al. 2012), was induced 154-fold. For downstream signaling, ABO3 and NACO19, which encode WRKY and NAC transcription factors (Jensen et al. 2010; Ren et al. 2010), were induced 32- and 20-fold, respectively. In addition, other ABA response-associated genes, includ- ing SDR4, PNP-A, 5PTASE11, SAP12, ARSK1, RLP41, UGT76B1, CRK36, LECRKA4.2, and ARCK1 (de Torres-Zabala et al. 2007; Ercetin and Gillaspy 2004; González-Guzmán et al. 2002; Hwang and Goodman 1995; Tanaka et al. 2012; von Saint Paul et al. 2011; Wang et al. 2008; Wang et al. 2011c; Xin et al.

Table 1. Classification and annotation of the differentially expressed genes $(P$ value $<0.01)$ involved in plant defense system

\begin{tabular}{|c|c|c|c|c|c|}
\hline \multirow[b]{2}{*}{ Category, name } & \multirow[b]{2}{*}{ AGI number b } & \multicolumn{2}{|c|}{ Base mean $^{a}$} & \multirow[b]{2}{*}{ Fold change $^{c}$} & \multirow[b]{2}{*}{ Functional annotations } \\
\hline & & $\beta$-estradiol & DMSO & & \\
\hline \multicolumn{6}{|l|}{$\overline{\text { PR genes }}{ }^{\mathrm{d}}$} \\
\hline PR1 & At2g14610 & $113,377.16$ & 95.893 & $1,182.3$ & Pathogenesis-related 1 \\
\hline$P R 2$ & At3g57260 & $2,443.0322$ & 24.506 & 99.691 & Pathogenesis-related 2 \\
\hline \multicolumn{6}{|l|}{ SA biosynthetic ${ }^{\mathrm{e}}$} \\
\hline EDS5 & At4g39030 & $3,487.6326$ & 120.4 & 28.967 & Enhanced disease susceptibility 5 \\
\hline SARD1 & At1g73805 & $6,853.2545$ & 295.14 & 23.221 & SAR deficient 1 \\
\hline SID2 & Atlg74710 & $4,121.1504$ & 157.69 & 26.134 & Salicylic acid induction deficient 2 \\
\hline CBP60g & At5g26920 & $3,950.3353$ & 263.17 & 15.01 & Calmodulin-binding protein $60 \mathrm{~g}$ \\
\hline \multicolumn{6}{|l|}{ SA accumulation ${ }^{\mathrm{f}}$} \\
\hline$A L D 1$ & At2g13810 & $3,410.672$ & 33.03 & 103.26 & AGD2-like defense response protein 1 \\
\hline WIN3 & At5g13320 & $11,246.583$ & 125.73 & 89.453 & HopW1-1-interacting 3 \\
\hline NUDX6 & At2g04450 & $7,305.6331$ & 85.238 & 85.708 & Nudix hydrolase homolog 6 \\
\hline FMO1 & At1g19250 & $8,314.5688$ & 23.441 & 354.71 & Flavin-dependent monooxygenase 1 \\
\hline PAD4 & At3g52430 & $11,497.174$ & 375.05 & 30.655 & Phytoalexin deficient 4 \\
\hline PDLP5 & At1g70690 & $1,842.3635$ & 98.024 & 18.795 & Plasmodesmata-located protein 5 \\
\hline \multicolumn{6}{|l|}{ Downstream $^{\mathrm{g}}$} \\
\hline WRKY51 & At5g64810 & $1,115.9298$ & 21.31 & 52.368 & WRKY transcription factor \\
\hline WRKY72 & At5g15130 & 652.28867 & 15.982 & 40.813 & WRKY transcription factor \\
\hline WRKY & At5g46350 & $1,080.2651$ & 31.964 & 33.796 & WRKY transcription factor \\
\hline WRKY38 & At5g22570 & 649.47303 & 33.03 & 19.663 & WRKY transcription factor \\
\hline WRKY50 & At5g26170 & 422.34518 & 17.048 & 24.774 & WRKY transcription factor \\
\hline \multicolumn{6}{|l|}{ Defense response $\mathrm{h}^{\mathrm{h}}$} \\
\hline DOX1 & At3g01420 & $11,457.755$ & 7.4584 & $1,536.2$ & Dioxygenase 1 \\
\hline$A I G 1$ & Atlg33960 & $38,678.372$ & 200.31 & 193.09 & AvrRpt2-induced gene 1 \\
\hline CML43 & At5g44460 & 136.089 & 1.0655 & 127.73 & Calmodulin like 43 \\
\hline PARG2 & At2g31865 & $1,982.2067$ & 44.75 & 44.295 & Poly (ADP-ribose) glycohydrolase 2 \\
\hline UGT85A1 & At1g22400 & $12,727.607$ & 438.98 & 28.994 & UDP-glucosyl transferase $85 \mathrm{~A} 1$ \\
\hline$T R X 5$ & At1g45145 & $7,811.5087$ & 312.19 & 25.022 & Cytosolic thioredoxin \\
\hline CDA1 & At2g19570 & $1,130.008$ & 58.601 & 19.283 & Cytidine deaminase 1 \\
\hline SDR3 & At2g47130 & $1,194.7676$ & 76.715 & 15.574 & Short-chain dehydrogenase/reductase 3 \\
\hline LOX1 & At1g55020 & $2,132.3739$ & 142.77 & 14.935 & Lipoxygenase 1 \\
\hline
\end{tabular}

a Base mean: the number of reads divided by the size factor (normalization constant) of sample. DMSO = dimethyl sulfoxide

${ }^{\mathrm{b}} \mathrm{AGI}=$ Arabidopsis Genome Initiative.

${ }^{\mathrm{c}}$ Fold change $=\beta$-estradiol base mean/DMSO base mean

${ }^{\mathrm{d}} \mathrm{PR}=$ pathogenesis related.

e Salicylic acid (SA) biosynthetic process.

${ }^{\mathrm{f}} \mathrm{SA}$ accumulation and signaling.

g Putative downstream signaling components.

${ }^{\mathrm{h}}$ Defense response-associated genes.

Table 2. Classification and annotation of the differentially expressed genes $(P<0.01)$ involved in resistance- or cell-death- related responses

\begin{tabular}{|c|c|c|c|c|c|}
\hline \multirow[b]{2}{*}{ Category, name } & \multirow[b]{2}{*}{ AGI number ${ }^{b}$} & \multicolumn{2}{|c|}{ Base mean $^{\mathbf{a}}$} & \multirow[b]{2}{*}{ Fold change $^{c}$} & \multirow[b]{2}{*}{ Functional annotations ${ }^{\mathrm{d}}$} \\
\hline & & $\beta$-estradiol & DMSO & & \\
\hline \multicolumn{6}{|c|}{ Resistance response ${ }^{\mathrm{e}}$} \\
\hline & At5g38344 & 172.69225 & 1.0654792 & 162.0794 & TIR domain family \\
\hline & At4g19925 & 119.1952 & 1.0654792 & 111.87 & TIR domain family \\
\hline & At1g57630 & $2,761.1989$ & 58.601355 & 47.11835 & TIR domain family \\
\hline & At2g20142 & $1,026.7681$ & 47.946563 & 21.41484 & TIR domain family \\
\hline & At5g38350 & 573.4509 & 1.0654792 & 538.2094 & Disease resistance protein (TIR-NBS-LRR class) \\
\hline & At4g11340 & 246.83729 & 1.0654792 & 231.6679 & Disease resistance protein (TIR-NBS-LRR class) \\
\hline & At5g18350 & 134.21191 & 1.0654792 & 125.9639 & Disease resistance protein (TIR-NBS-LRR class) \\
\hline & At3g04220 & 309.7198 & 12.78575 & 24.22383 & Disease resistance protein (TIR-NBS-LRR class) \\
\hline \multicolumn{6}{|l|}{ Cell death } \\
\hline$B A P 2$ & At2g45760 & $2,243.1222$ & 2.1309584 & $1,052.635$ & Similar to BONZAI1-binding protein (BAP1) \\
\hline$K T I 1$ & At1g73260 & $4,257.2394$ & 10.654792 & 399.561 & Kunitz trypsin inhibitor 1 (KTI1) \\
\hline BON1 & At5g61900 & $2,020.6871$ & 106.54792 & 18.96505 & Encodes a copine-like protein \\
\hline BON3 & At1g08860 & 101.36284 & 2.1309584 & 47.56679 & Encodes a copine-like protein \\
\hline$B A P 1$ & At3g61190 & $1,154.4102$ & 51.143001 & 22.5722 & BON ASSOCIATION PROTEIN 1 \\
\hline
\end{tabular}

a Base mean: the number of reads divided by the size factor (normalization constant) of sample. DMSO = dimethyl sulfoxide.

${ }^{\mathrm{b}}$ AGI = Arabidopsis Genome Initiative.

${ }^{\mathrm{c}}$ Fold change $=\beta$-estradiol base mean/DMSO base mean.

${ }^{\mathrm{d}} \mathrm{TIR}=$ Toll-interleukin-resistance, NBS-LRR = nucleotide-binding site leucine-rich repeat. 
2009), were also highly expressed. Among them, SDR4, which encodes a short-chain dehydrogenase/reductase that may participate in ABA synthesis, was induced 128-fold; $P N P-A$, which encodes a plant natriuretic peptide that modulates the effects of ABA, was induced 88-fold; and UGT76B1, which encodes a glucosyltransferase that attenuates SA-mediated defense system, was found to contain a ABA response element in the promoter. These results suggest that $X$. campestris pv. campestris 8004 may interfere plant ABA signaling transduction by AvrXccC $\mathrm{C}_{8004}$.

To validate the RNA-Seq profiling on the differentially expressed genes induced by $\mathrm{AvrXccC}_{8004}$ (110 to 440 aa), quantitative real-time reverse-transcription polymerase chain reaction (qRT-PCR) was used. Nine genes involved in plant defense responses, especially in the SA-signaling pathway, including biosynthetic process, accumulation, signaling transduction, and downstream response, were evaluated using $E F 1 \alpha$ as a reference gene. PR1, EDS5, SID2, WIN3, FMO1, PAD4, WRKY51, DOX1, and $A I G 1$ were upregulated after $\beta$-estradiol treatment (Fig. 3A). At the same time, NCED5, SAG113, PDR12, ABO3, NAC019, $P N P-A$, and SAP12, which are involved in ABA biosynthesis, ABA signaling transduction, and ABA regulated response, were also evaluated using $E F 1 \alpha$ as a reference gene. Similar to the examined genes involved in plant defense responses, all ABAsignaling response genes were upregulated after $\beta$-estradiol treatment (Fig. 3B). Here, our qRT-PCR results were consistent with the high-throughput gene expression profiling produced by next-generation sequencing.

To further examine whether $\mathrm{AvrXccC}_{8004}$ (1 to 440 aa) triggered molecular responses similar to $\mathrm{AvrXccC}_{8004}$ (110 to 440 aa), all of the above-validated genes were investigated again by qRT-PCR using RNA samples extracted from $X V E: A v r X c c C_{8004}$ (1 to 440 aa) transgenic plants. Compared with the DMSO control, all examined genes involved in plant defense responses and ABA-signaling responses were upregulated upon $\beta$-estradiol treatment (Fig. 3C and D). However, the relative expression ratios of examined genes induced by $\mathrm{AvrXccC}_{8004}$ (1 to 440 aa) were not as high as those induced by $\mathrm{AvrXccC}_{8004}(110$ to $440 \mathrm{aa}$ ), although the transcript levels and protein amounts of $A v r X c c C_{8004}$ (110 to 440 aa) were only slightly higher than those of $A v r X c c C_{8004}$ (1 to 440 aa) in Arabidopsis after $\beta$ estradiol treatment (Fig. 3E and F).

\section{AvrXccC of $X$. campestris pv. campestris 8004 is required to manipulate $A B A$ biosynthesis and signaling.}

The highly induced NCED5, which encodes a key regulatory enzyme in the $\mathrm{ABA}$ biosynthetic process, prompted us to examine whether $\mathrm{AvrXccC} \mathrm{C}_{8004}$ could manipulate the accumulation of ABA. ABA amounts were induced 2.4- and 3.0-fold upon $\beta$-estradiol treatment in $X V E: A v r X c c C_{8004}$ (1 to 440 aa) and $X V E: A v r X c c C_{8004}(110$ to 440 aa) transgenic plants, respectively (Fig. 4A). We further examined the ABA amounts and ABA-related gene expression levels in $X$. campestris pv. campestris 8004 wild-type (WT)- and $\triangle a v r X c c C$-challenged leaves. The $\Delta a v r X c c C$ mutant was severely impaired in the ability to manipulate the accumulation of ABA in challenged leaves (Fig. 4B). Consistently, the expression levels of NCED5, $S A G 113, A B O 13$, and NACO19 involved in ABA biosynthesis, accumulation, and signaling were dramatically reduced in leaves challenged by the $\Delta a v r X c c C$ mutant (Fig. 4C).

\section{Exogenous application of ABA enhances the multiplication of $X$. campestris pv. campestris 8004.}

In order to test the effect of exogenous ABA on the growth of $X$. campestris pv. campestris 8004, ABA was sprayed on Arabidopsis leaves $24 \mathrm{~h}$ before bacterial inoculation. Followed ABA pretreatment, $X$. campestris pv. campestris 8004 strains, including the WT and $\Delta a v r X c c C$ mutant, were inoculated into Arabidopsis leaves. Exogenous application of ABA increased the populations of $X$. campestris pv. campestris 8004 strains by more than two orders of magnitude at 3 days post inoculation compared with mock $(0.2 \%$ ethanol) treatment (Fig. 5A and B). Thus, the exogenous application of ABA allowed $X$. campestris pv. campestris 8004 strains to partially overcome host defense responses. These results indicate that ABA is a key factor in regulating the interactions of $X$. campestris pv. campestris and Arabidopsis.

Table 3. Classification and annotation of the differentially expressed genes $(P<0.01)$ involved in abscisic acid (ABA) responses

\begin{tabular}{|c|c|c|c|c|c|}
\hline \multirow[b]{2}{*}{ Category, name } & \multirow[b]{2}{*}{ AGI number ${ }^{b}$} & \multicolumn{2}{|c|}{ Base mean $^{\mathrm{a}}$} & \multirow[b]{2}{*}{ Fold change $^{c}$} & \multirow[b]{2}{*}{ Functional annotations } \\
\hline & & $\beta$-estradiol & DMSO & & \\
\hline \multicolumn{6}{|l|}{$\overline{\text { ABA biosynthetic }}{ }^{\mathrm{d}}$} \\
\hline NCED5 & At1g30100 & 160.491 & 1.06548 & 150.63 & 9-cis-Epoxycarotenoid dioxygenase \\
\hline \multicolumn{6}{|c|}{ ABA accumulation ${ }^{\mathrm{e}}$} \\
\hline SAG113 & At5g59220 & 492.736 & 3.19644 & 154.15 & Senescence associated gene 113 \\
\hline PDR12 & Atlg15520 & $12,631.9$ & 148.102 & 85.292 & Pleiotropic Drug resistance 12 \\
\hline \multicolumn{6}{|l|}{ Downstream ${ }^{\mathrm{f}}$} \\
\hline ABO3 & At1g66600 & 370.725 & 11.7203 & 31.631 & ABA Overly sensitive mutant 3 \\
\hline NACO19 & At1g52890 & $1,615.24$ & 79.9109 & 20.213 & NAC transcription factor \\
\hline \multicolumn{6}{|l|}{ ABA response ${ }^{g}$} \\
\hline SDR4 & At3g29250 & 409.206 & 3.19644 & 128.02 & Short-chain dehydrogenase/reductase 4 \\
\hline$P N P-A$ & At2g18660 & $2,987.39$ & 34.0953 & 87.619 & Plant natriuretic peptide A \\
\hline 5PTASE11 & At1g47510 & 305.966 & 3.19644 & 95.721 & Inositol polyphosphate 5-phosphatase 11 \\
\hline SAP12 & At3g28210 & 838.121 & 21.3096 & 39.331 & Stress-associated protein 12 \\
\hline ARSKI & At2g26290 & 222.435 & 6.39288 & 34.794 & Arabidopsis root-specific kinase 1 \\
\hline RLP41 & At3g25010 & $1,590.83$ & 55.4049 & 28.713 & Receptor like protein 41 \\
\hline UGT76B1 & At3g11340 & $1,755.08$ & 67.1252 & 26.146 & Glucosyltransferase \\
\hline CRK36 & At4g04490 & $2,588.51$ & 122.53 & 21.125 & Cysteine-rich receptor like protein kinase 36 \\
\hline LECRKA4.2 & At5g01550 & $1,001.43$ & 54.3394 & 18.429 & Lectin receptor kinase $\mathrm{A} 4.2$ \\
\hline ARCKI & At4g11890 & 905.696 & 42.6192 & 21.251 & ABA- and osmotic stress-inducible receptor-like cytosolic kinase 1 \\
\hline
\end{tabular}

\footnotetext{
${ }^{\mathrm{b}}$ AGI $=$ Arabidopsis Genome Initiative.

${ }^{\mathrm{c}}$ Fold change $=\beta$-estradiol base mean/DMSO base mean.

d ABA biosynthetic process.

${ }^{\mathrm{e}} \mathrm{ABA}$ accumulation and signaling.

${ }^{\mathrm{f}}$ Downstream signaling components.

g ABA response-associated genes.
}

${ }^{\mathrm{a}}$ Base mean: the number of reads divided by the size factor (normalization constant) of sample. DMSO = dimethyl sulfoxide. 


\section{DISCUSSION}

In Arabidopsis, the effects of ABA in regulating plantpathogen interactions have been intensively studied in recent years (Asselbergh et al. 2008). In P. syringae, it has been demonstrated that the manipulations of host ABA biosynthesis and response machinery were mainly controlled by the virulence effectors (de Torres-Zabala et al. 2007). Here, in X. campestris pv. campestris, $\mathrm{AvrXccC} \mathrm{C}_{8004}$ not only presented its avirulence activity to trigger plant defense responses but also possessed its virulence activity to manipulate the components involved in the ABA signaling pathway, leading to an increase of ABA concentrations and disturbance of ABA signaling responses. Our data suggest that the increase of ABA levels was caused by the highly expressed NCED5. NCED5 not only encodes a key enzyme involved in ABA biosynthesis (Frey et al. 2012) but also plays an important role in regulating plant defense

A

Defense response (SA-mediated signaling)

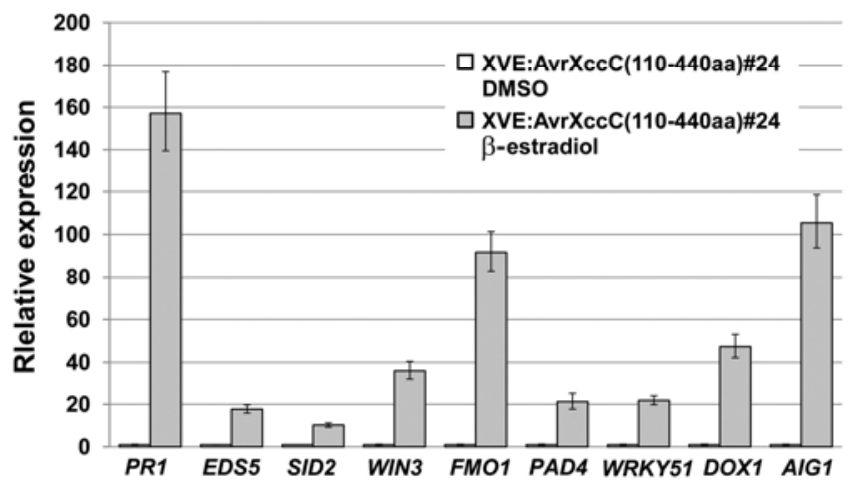

C

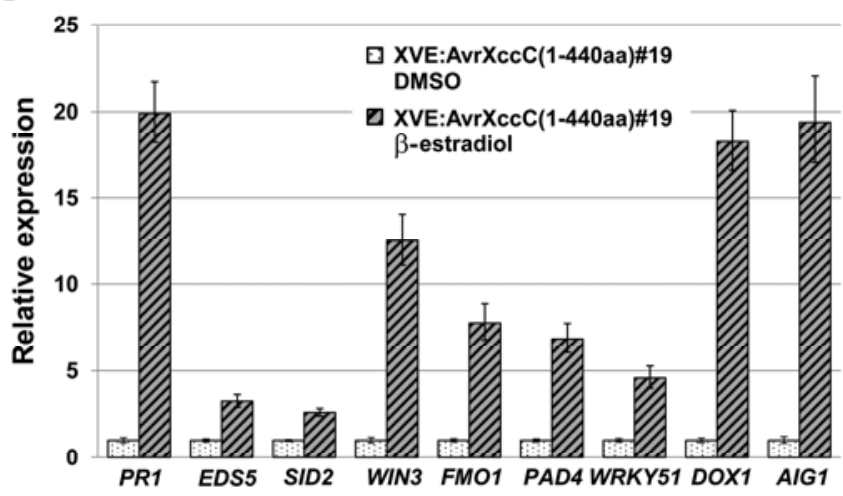

responses because the $c d s 2-1 D$ mutant, an activation-tagged line with highly expressed NCED5, showed the enhanced disease susceptibility phenotype (Fan et al. 2009). As a result, $\mathrm{AvrXccC}_{8004}$ may attenuate host defense responses by elevating ABA levels. This hypothesis was supported by our findings on exogenous application of ABA, which enhanced the multiplication of $X$. campestris pv. campestris in Arabidopsis. Recently, Choi and Hwang (2011) also showed that ABR1, a negative regulator of $\mathrm{ABA}$ biosynthesis, was required for the resistant pepper to protect against $X$. campestris pv. vesicatoria. Taken together, it is clear that ABA functions as a negative signaling molecule to suppress host immunity required to protect against Xanthomonas spp. Although ABA plays an important role in modulating the pathogenicity of Xanthomonas spp., the molecular mechanisms of ABA actions in plant defense pathways are still unclear and warrant further investigation.
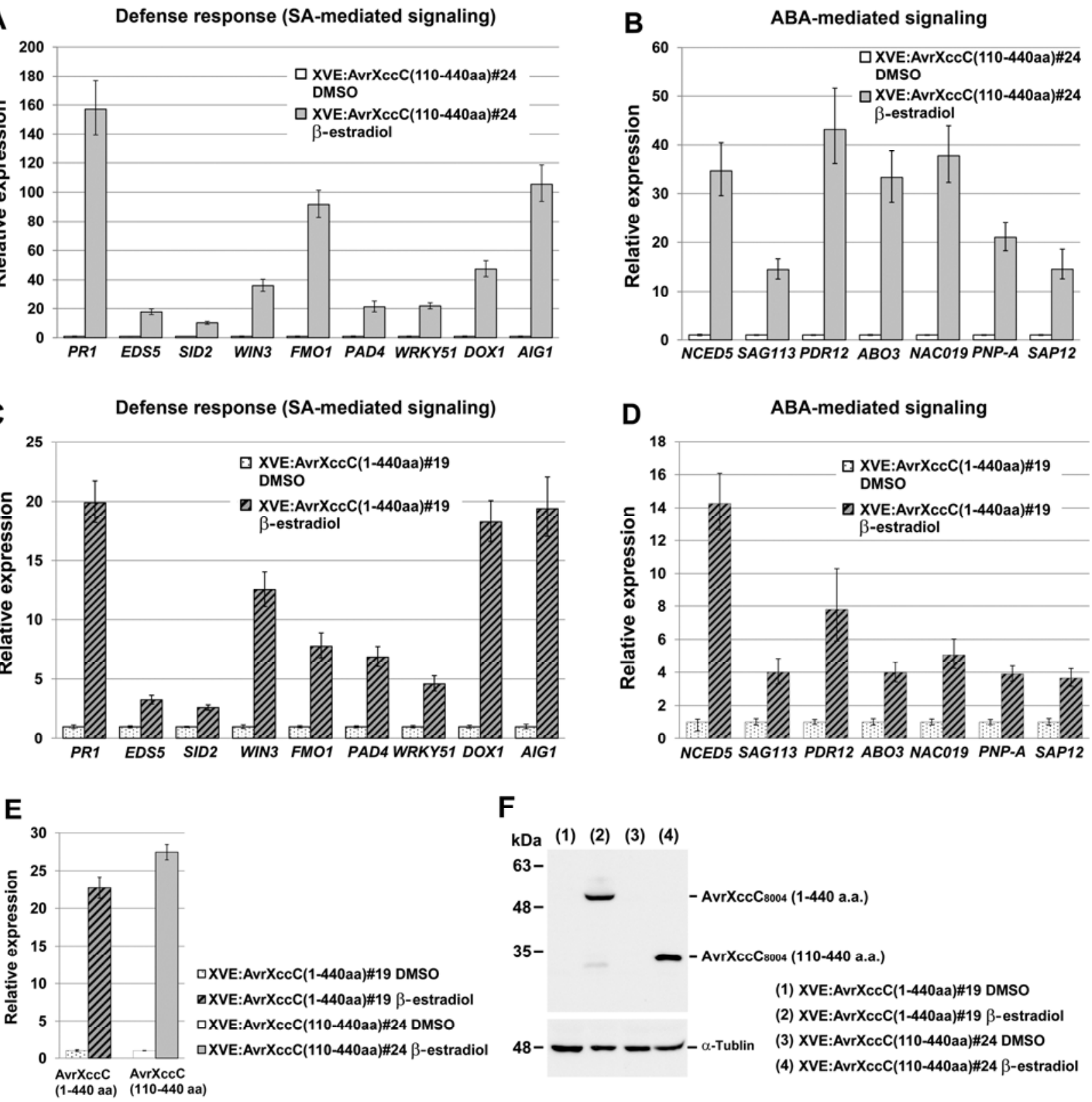

$\mathbf{F}$

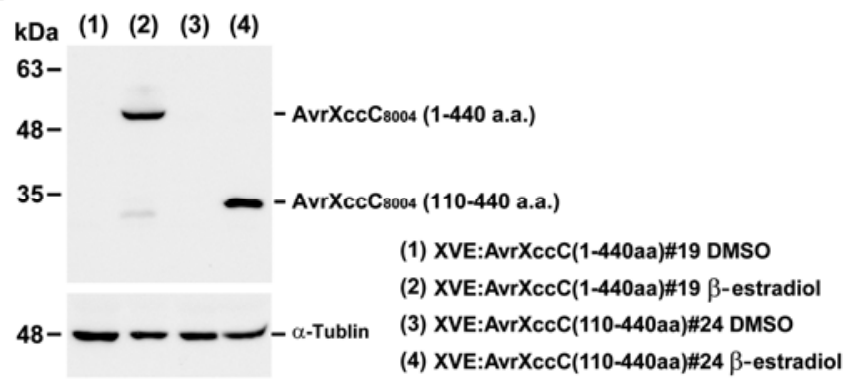

Fig. 3. Quantitative reverse-transcription polymerase chain reaction (qRT-PCR) validation of differentially expressed genes. mRNA levels of genes involved in A and $\mathbf{C}$, plant defense responses and $\mathbf{B}$ and $\mathbf{D}$, abscisic acid (ABA) signaling responses were normalized with $E F 1 \alpha$, and the relative expression levels in a dimethyl sulfoxide (DMSO) control were set at 1 for each gene. E, Relative expression levels of $A v r X c c C_{8004}\left(110\right.$ to 440 amino acids [aa]) and $A v r X c c C_{8004}(1$ to 440 aa) were examined by qRT-PCR and $\mathbf{F}$, the translated products of those transcripts were examined by Western blotting using specific polyclonal antibody against $\mathrm{AvrXcc}_{8004}(110$ to $440 \mathrm{aa})$. Anti- $\alpha$-tubulin was used for loading control. Average and standard error values of three independent repeats are given. 
In Arabidopsis, there are 149 NBS-LRR-encoding genes that can be divided into two distinct groups: TIR-NBS-LRR and coiled-coil motif (CC)-NBS-LRR, which play an important role in pathogen sensing and host defense (DeYoung and Innes 2006; Meyers et al. 2003). Early studies demonstrated that TIR-NBS-
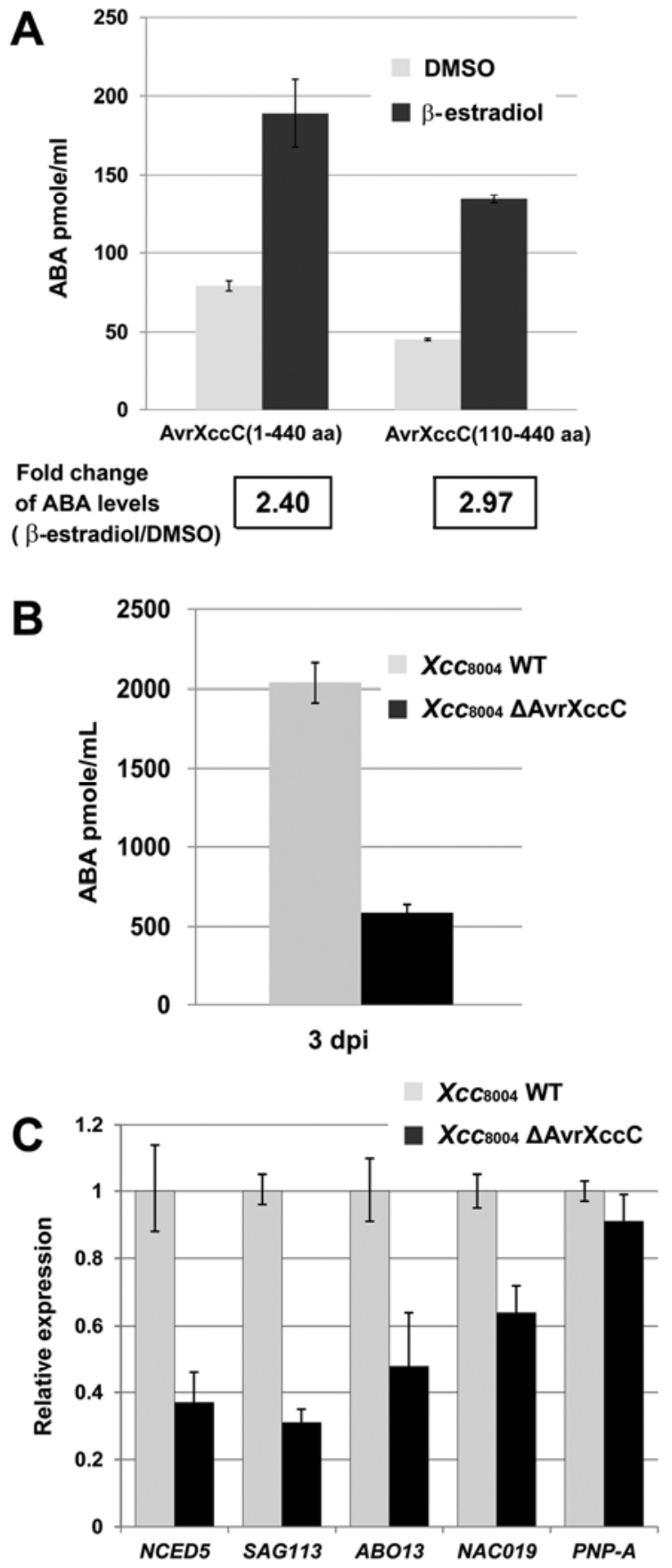

Fig. 4. AvrXccC of Xanthomonas campestris pv. campestris 8004 is required to manipulate abscisic acid (ABA) biosynthesis and signaling. A, Quantification of ABA levels in Arabidopsis plants expressing AvrXcc $\mathrm{C}_{8004}$ (1 to 440 amino acids [aa]) and $\operatorname{AvrXccC}_{8004}$ (110 to 440 aa). ABA levels in Arabidopsis transgenic plants were determined by a phytodetek ABA enzyme-linked immunosorbent assay kit and the fold changes in ABA levels after $\beta$-estradiol treatment were shown in boxes. B, Quantification of ABA levels in $X$. campestris pv. campestris 8004 wild-type (WT)- and $\triangle a v r X c c C$-challenged leaves. ABA levels in challenged leaves were determined at 3 days post inoculation (dpi). C, Examination of ABA-related gene expression levels in $X$. campestris pv. campestris 8004 WT- and $\triangle a v r X c c C$-challenged leaves by quantitative reverse-transcription polymerase chain reaction. Average and standard error values of three independent repeats are given.
LRR proteins and CC-NBS-LRR proteins mounted different signaling pathways for R-mediated resistant responses (Aarts et al. 1998; Głowacki et al. 2011). For the TIR-NBS-LRR proteinmediated signaling pathway, EDS1 played a key role as a mediator which interacted with PAD4 to regulate the levels of SA (Feys et al. 2001). The activity of EDS1 was also regulated by a monooxygenase, FMO1 (Bartsch et al. 2006). For CC-NBSLRR protein-mediated signaling pathways, NDR1 played a key role as a mediator to trigger resistance responses. The RNA-Seq profiling assay obtained here revealed that only genes encoding TIR-NBS-LRR proteins were highly upregulated, which suggests that the AvrB_AvrC domain of AvrXccC ${ }_{8004}$ may trigger EDS1-mediated resistance responses. Moreover, the highly expressed PAD4 and FMO1 induced by $\mathrm{AvrXccC}_{8004}$ (110 to 440 a) further support the idea. Because no $\mathrm{R}$ protein responsible for the type III effectors of $X$. campestris pv. campestris 8004 was identified thus far, it will be interesting to isolate the corresponding $R$ gene, which should provide invaluable insights into the molecular mechanism of the effector-triggered immunity signaling pathway in Arabidopsis.

In this study, we showed that the intact AvrB_AvrC domain of $\mathrm{AvrXccC}_{8004}$ is essential and sufficient to elicit defense responses, because the dissection of $\mathrm{AvrXccC}_{8004}$ (110 to $\left.440 \mathrm{aa}\right)$ into three subdomains-AvrXccC $8004 \quad(110$ to 237aa), $\operatorname{AvrXccC}_{8004}$ (238 to 338 aa), and $\mathrm{AvrXccC}_{8004}$ (339 to 440 aa) - fully disrupts its avirulence functions. These results suggest that the functional activities of the AvrB_AvrC domain of $\mathrm{AvrXccC}_{8004}$ are necessary to be present for the recognition by the host R-mediated defense system more than structural or
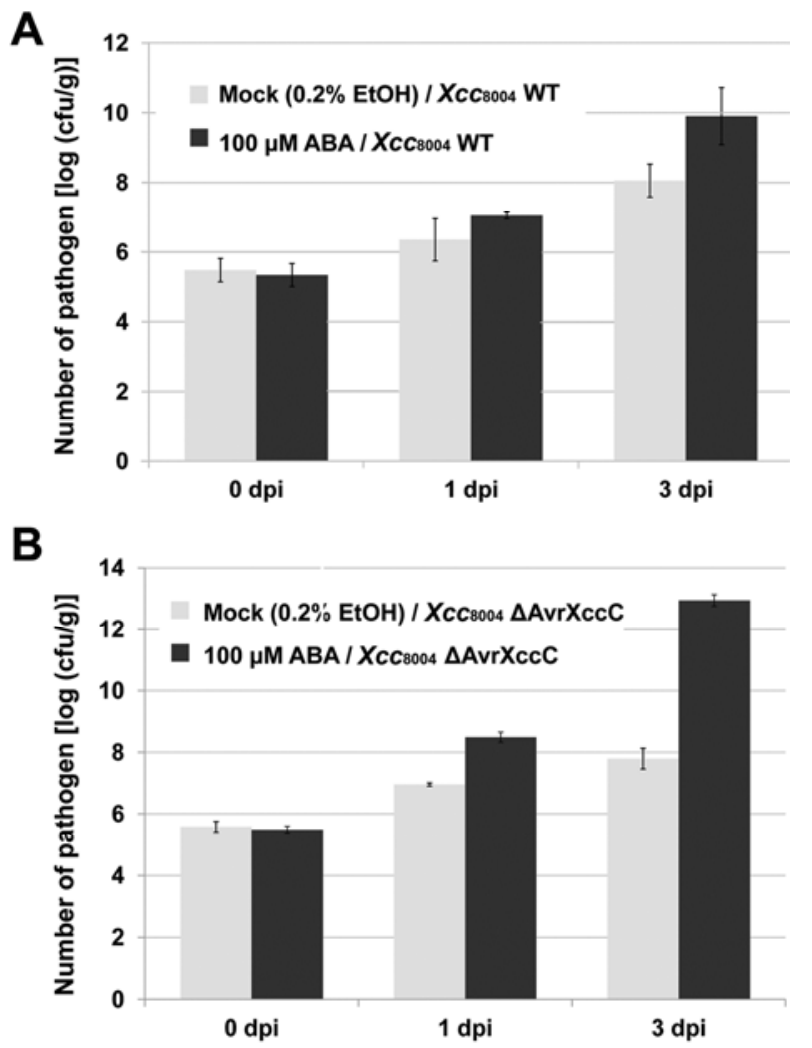

Fig. 5. Effect of exogenous application of abscisic acid (ABA) on the growth of Xanthomonas spp. in wild-type (WT) Arabidopsis. Multiplication of A, Xanthomonas campestris pv. campestris 8004 WT and B, $\triangle a v r X c c C$ mutant strains on Arabidopsis plants sprayed with ABA. Exogenous $100 \mu \mathrm{M}$ ABA or $0.2 \%$ ethanol were applied on Arabidopsis $24 \mathrm{~h}$ before bacterial inoculation. Hand-infiltrated leaves were collected at indicated times for measuring in planta growth of bacterial populations. Average and standard error values of three independent repeats are given. 
sequential presentations. Recently, with bioinformatic predictions, it has been suggested that AvrXccC contains a presumable fido domain, which may be able to modify host proteins by AMPylation (Kinch et al. 2009). However, it remains to be determined whether $\mathrm{AvrXccC}_{8004}$ can possess its functional activities through AMPylation to modify host proteins which, in turn, modulate host defense responses.

Previous studies showed that the N-terminal myristoylation of $\mathrm{AvrXccC}_{8004}$ was essential for membrane localization and the myristoylation site mutation dramatically destroyed the avirulence activity of $\mathrm{AvrXccC}_{8004}$ (Wang et al. 2007). In this study, we found that overexpression of $\mathrm{AvrXccC}_{8004}$ (110 to 440 aa) as well as $\mathrm{AvrXccC}_{8004}$ (1 to $440 \mathrm{aa}$ ) was able to trigger plant resistance responses and caused cell death in Arabidopsis. To clarify the subcellular localization of $\mathrm{AvrXccC}_{8004}$ (110 to 440 aa) in Arabidopsis, C-terminal yellow fluorescent protein (YFP)-tagged $\mathrm{AvrXccC}_{8004}$ (110 to 440 aa) was expressed transiently in mesophyll protoplast. Similar to the localization pattern of full-length $\mathrm{AvrXccC}_{8004}$ with a deleted Nterminal myristoylation site (Wang et al. 2007), the $\mathrm{AvrXccC}_{8004}$ (110 to 440 aa) lost the ability for plasma membrane targeting (Supplementary Fig. S4). However, instead of even distribution in onion epidermis cells for myristoylation site-mutated AvrXccC ${ }_{8004}$-YFP (Wang et al. 2007), the fluorescence signals of $\mathrm{AvrXccC}_{8004}$ (110 to 440 aa)-YFP were observed as cytoplasmic foci in Arabidopsis protoplasts. Although the cytoplasmic localization of $\mathrm{AvrXccC}_{8004}$ (110 to 440 aa) did not abolish its avirulence activity, it is still unclear how $\mathrm{AvrXccC}_{8004}$ (110 to 440 aa) was recognized and triggered resistance responses. Hence, the identification of $\mathrm{AvrXccC}_{8004}$ targets or host $\mathrm{R}$ proteins will clarify how the cytoplasmic foci formed by $\mathrm{AvrXccC}_{8004}$ (110 to 440 aa) responsible for the resistance responses.

\section{Conclusion.}

$X$. campestris pv. campestris type III effector AvrXccC $\mathrm{C}_{8004}$ has been characterized as a bifunctional effector, which induced strong defense responses in WT Arabidopsis Col-0 and acted as virulence factor in rarl and sgtlb mutants (Rong et al. 2010). In this study, we further characterized the functions of $\mathrm{AvrXccC}_{8004}$ using Arabidopsis protoplast and transgenic approaches. We found that the AvrB_AvrC domain of $\mathrm{AvrXccC}_{8004}$ was essential and sufficient to elicit defense responses, because both $\operatorname{AvrXccC}_{8004}$ (1 to 440 aa) and AvrXccC $_{8004}$ (110 to 440 aa) induced similar physiological phenotypes as well as molecular phenotypes. Remarkably, we showed that the manipulation of $\mathrm{ABA}$ biosynthesis and response machinery by $\mathrm{AvrXccC}_{8004}$ is an important strategy to attenuate host defense responses. However, as an avirulence factor, $\mathrm{AvrXccC} \mathrm{C}_{804}$ elicited strong R-mediated resistance responses, mainly through the SA-signaling pathway, which suppressed the antagonistic functions of ABA activated by the virulence activities of $\mathrm{AvrXccC} \mathrm{C}_{8004}$. As a result, cell death, the typical outcome of hypersensitive response, was observed in both $X V E: A v r X c c C_{8004}$ (1 to 440 aa) and $X V E: A v r X c c C_{8004}$ (110 to 440 aa) transgenic plants upon $\beta$-estradiol treatment.

\section{MATERIALS AND METHODS}

\section{Plant materials, growth conditions, and $\beta$-estradiol treatments.}

Arabidopsis ecotype Col-0 was used for protoplast preparation, transgenic plant generation, and Xanthomonas spp. inoculation. Plants were grown at $21^{\circ} \mathrm{C}$ in a semi-controlled walkin chamber with a photoperiod of 16 and $8 \mathrm{~h}$ at 8,000 lux for Agrobacterium transformation, and a photoperiod of 12 and 12 $\mathrm{h}$ at 8,000 lux for protoplast preparation and Xanthomonas spp. inoculation. For trypan blue staining, TEM investigation, quantitative real-time RT-PCR, ABA measurement, and nextgeneration sequencing analysis, 4- to 5-week-old transgenic plants were sprayed with $20 \mu \mathrm{M} \beta$-estradiol (Sigma-Aldrich, St. Louis) every $12 \mathrm{~h}$ for $48 \mathrm{~h}$ before harvest.

\section{Constructions.}

DNA fragments encoding full-length and N-terminal-truncated AvrXccC (XC_2004) were amplified from X. campestris pv. campestris 8004 genomic DNA by PCR using AccuPrime pfx DNA polymerase (Invitrogen, Carlsbad, CA, U.S.A.). For protoplast transient expression analysis, PCR products were subcloned into pWEN18 vector by restriction site reconstructions to generate C-terminal YFP-tagged constructs under the control of Cauliflower mosaic virus $35 \mathrm{~S}$ promoter. For generation of transgenic plants, PCR products were subcloned into pER8 vector under the control of the XVE promoter (Zuo et al. 2000) by restriction site reconstructions. All plasmids were verified by DNA sequencing. Primer sequences are listed in Supplementary Table S6.

\section{Arabidopsis protoplasts analysis.}

To observe subcellular localizations of YFP-tagged effectors, Arabidopsis mesophyll protoplasts were prepared according to Yoo and associates (2007). Briefly, leaves from 4- to 5-week-old plants grown in a relatively short photoperiod were used. Cellulase R10 and Macerozyme R10 obtained from Yakult Pharmaceutical Ind. Co. were prepared for enzyme solution to digest leaves. Plasmid DNAs were purified by plasmid Maxi kit (Qiagen, Hilden, Germany) according to the manufacturer's instructions. After digestion and wash, fresh protoplasts were transfected with $10 \mu \mathrm{g}$ of plasmid DNAs using the polyethylene glycol-calcium method. After $12 \mathrm{~h}$ of incubation at room temperature, subcellular localizations of YFP-tagged effectors were observed by confocal laser-scanning microscopy and images were collected with the Olympus fluoview FV1000 system.

\section{Arabidopsis transformations.}

To obtain Arabidopsis transgenic plants expressing $\operatorname{AvrXccC}_{8004}\left(1\right.$ to 440 aa) and $\operatorname{AvrXccC}_{8004}$ (110 to 440 aa), plasmid DNAs were introduced into A. tumefaciens strain ABI or EHA105 by the freeze-thaw method (Weigel and Glazebrook 2006). Agrobacterium-mediated transformation of Arabidopsis Col-0 plants was performed using the floral-dip method (Clough and Bent 1998). After transformation, seed were collected and selected on half-strength Murashige and Skoog $(1 / 2 \times \mathrm{MS})$ medium containing hygromycin $(12.5 \mu \mathrm{g}$ $\mathrm{ml}^{-1}$ ) and carbencillin $\left(100 \mu \mathrm{g} \mathrm{ml}^{-1}\right)$. For morphological examination, homozygous seed were obtained and germinated on $1 / 2 \times \mathrm{MS}$ medium containing $20 \mu \mathrm{M} \beta$-estradiol. For transcriptome analysis, homozygous seed were grown on soil before $\beta$-estradiol treatment.

\section{Trypan blue staining.}

For the cell death assay, leaves were detached and stained with trypan blue according to van Wees (2008). Briefly, leaves were boiled in trypan blue-lactophenol solution $(10 \mathrm{ml}$ of lactic acid, $10 \mathrm{ml}$ of glycerol, $10 \mathrm{~g}$ of phenol, and $10 \mathrm{mg}$ of trypan blue dissolved in $10 \mathrm{ml}$ of distilled water) for $1 \mathrm{~min}$, followed by overnight incubation at room temperature. After staining, samples were destained with chloral hydrate $\left(2.5 \mathrm{~g} \mathrm{ml}^{-1}\right)$ until the background was clear. Images were collected with Leica ZM75 microscopy.

\section{Northern blotting.}

For Northern blotting analysis, transgenic seed were germinated on $1 / 2 \times$ MS medium. Seedlings ( 2 to 3 weeks old) were 
treated with DMSO or $20 \mu \mathrm{M} \beta$-estradiol for $12 \mathrm{~h}$. Total RNA was extracted using Trizol reagent (Invitrogen) according to the manufacturer's instructions. Total RNA $(12 \mu \mathrm{g})$ was fractionated on $1.2 \%$ (wt/vol) agarose gel and then transferred to Hybond-XL membrane (GE Bioscience, Piscataway, NJ, U.S.A.). To detect transcripts of a deletion series of $A v r X c c C_{8004}$, the coding region of the $A v r X c c C_{8004}$ gene was amplified and labeled with $\left[\alpha_{-}{ }^{32} \mathrm{P}\right] \mathrm{dCTP}$ using the random prime labeling system (GE Bioscience). Hybridization was performed overnight at $65^{\circ} \mathrm{C}$ in hybridization buffer $(0.3 \mathrm{M}$ sodium phosphate [pH 7.0], $10 \mathrm{mM}$ EDTA, 5\% [wt/vol] sodium dodecyl sulfate [SDS], $10 \%$ [wt/vol] dextran sulfate, and salmon sperm DNA at $\left.0.15 \mathrm{mg} \mathrm{m}^{-1}\right)$, and then washed with $0.1 \times \mathrm{SSC}(1 \times \mathrm{SSC}$ is $0.15 \mathrm{M} \mathrm{NaCl}$ plus $0.015 \mathrm{M}$ sodium citrate) and $0.1 \%$ (wt/vol) $\mathrm{SDS}$ at $65^{\circ} \mathrm{C}$. Signals were detected by autoradiography.

\section{TEM.}

For ultrastructural analysis, leaves were detached and cut into transverse sections less than $2 \mathrm{~mm}$ wide. Samples were fixed with $2.5 \%$ (wt/vol) glutaraldehyde in $0.1 \mathrm{M}$ phosphate buffer overnight at $4{ }^{\circ} \mathrm{C}$ and then post-fixed with $1 \%$ (wt/vol) $\mathrm{OsO}_{4}$. After being dehydrated in a graded series of acetone, samples were embedded in Spurr's resin mixture and cut by glass knives on an ultramicrotome. Ultrathin sections $(70 \mathrm{~nm})$ were collected with uncoated copper grids (75-mesh square) and stained with uranyl acetate and lead citrate. Sections were examined with a JOEL JEM-1400 transmission electron microscope at an acceleration voltage of $100 \mathrm{KeV}$. Images were collected with a digital camera.

\section{Next-generation sequencing.}

For RNA-Seq analysis, total RNA was extracted using the RNeasy plant mini kit (Qiagen) according to the manufacturer's instructions. RNA quality was determined using $1.2 \%$ (wt/vol) formaldehyde gel electrophoresis and an Experion RNA analysis kit (Bio-Rad, Munich). For Experion RNA analysis, RNA samples with an RNA quality indicator number greater than 9.3 were presented at each sample. Next-generation sequencing was done using Illumina Hiseq 2000. Sequence reads were aligned using CLC-bio. Differentially expressed genes were identified by DESeq. Gene expression levels were normalized as RPKM. GO analysis was performed by Blast2GO.

\section{qRT-PCR.}

For RNA expression analysis, leaves were collected and total RNA was extracted using Trizol reagent (Invitrogen) according to the manufacturer's instructions. cDNAs were synthesized by reverse transcription using Superscript III first-strand synthesis supermix (Invitrogen). qRT-PCR was performed using the KAPA SYBR fast qPCR kit (Kapa Biosystems, Boston) and Illumina Eco real-time PCR system to validate the RNA-Seq results. Expression levels of selected genes were normalized to the expression of reference gene EFl $\alpha$. Experiments were repeated at least three times.

\section{Antibody production.}

A DNA fragment encoding $A v r X c c C_{8004}$ (110 to 440 aa) was amplified by PCR using AccuPrime Pfx DNA polymerase (Invitrogen). The PCR product was subcloned into pETSUMO (Invitrogen) and verified by DNA sequencing. Recombinant protein $\mathrm{His}_{6}-\mathrm{SUMO}-\mathrm{AvrXccC} \mathrm{C}_{8004}(110$ to 440 aa) was produced in Escherichia coli BL21 (DE3) cells and purified using $\mathrm{Ni}^{2+}$-NTA resin (Qiagen) according to the manufacturer's instructions. After being cleaved with Ulp1 to remove the $\mathrm{His}_{6-}$ SUMO tag, recombinant protein $\mathrm{AvrXccC}_{8004}$ (110 to 440 aa) was purified for antibody production in rabbit. Specific poly- clonal antibodies against $\mathrm{AvrX} \mathrm{XcC}_{8004}$ were obtained by affinity purification using polyvinylidene diflouride membrane as a coupling matrix.

\section{Measurement of endogenous ABA levels.}

The extraction and measurement of endogenous ABA were performed following the method described by Hsu and associates (2011). After $\beta$-estradiol treatment, aerial parts of transgenic plants were collected and frozen immediately in liquid nitrogen. The frozen samples were lyophilized and their dry weights were measured. To measure the endogenous ABA content, 0.1-g dried samples were extracted with solution containing $80 \%$ (vol/vol) methanol and $2 \%$ (vol/vol) glacial acetic acid in the dark at $4^{\circ} \mathrm{C}$. After overnight extraction on a rotary shaker, samples were centrifuged and the clear supernatants were dried with a speedvac concentrator. After being resuspended in Tris-buffered saline buffer, the endogenous ABA levels were determined with a Phytodetek ABA enzyme immunoassay test kit (Agdia, Elkhart, IN, U.S.A.) according to the manufacturer's instructions. Briefly, reactions were incubated at $37^{\circ} \mathrm{C}$ for $2 \mathrm{~h}$, and the absorbance values were read at $405 \mathrm{~nm}$ by an enzyme-linked immunosorbent assay reader. Experiments were repeated at least three times.

\section{ABA treatment and bacterial inoculation.}

ABA treatment was performed following the method described by de Torres-Zabala and associates (2007). X. campestris pv. campestris 8004 strains were cultured in NY broth ( $3 \mathrm{~g}$ of beef extract, $5 \mathrm{~g}$ of peptone, and $3 \mathrm{~g}$ of yeast extract in 1 liter of water) at $28^{\circ} \mathrm{C}$. Arabidopsis Col-0 plants (4 to 5 weeks old) were used for bacterial growth assay. At $24 \mathrm{~h}$ after plants were sprayed with $100 \mu \mathrm{M}$ ABA (Acros, Waltham, MA, U.S.A.), leaves were infiltrated with a bacterial suspension $(2 \times$ $10^{6} \mathrm{CFU} \mathrm{ml} \mathrm{m}^{-1}$ ) by a syringe. The inoculated plants were kept at $22^{\circ} \mathrm{C}$ in a growth chamber. Bacterial populations in leaves were determined at indicated time intervals using NY agar medium containing rifampicin $\left(50 \mu \mathrm{g} \mathrm{ml}^{-1}\right)$. Control assays were done by using plants sprayed with a solution of $0.2 \%$ ( $\mathrm{vol} / \mathrm{vol})$ ethanol. Experiments were repeated at least three times.

\section{ACKNOWLEDGMENTS}

We thank M. Dow for providing us with $X$. campestris pv. campestris 8004 strain; W.-N. Jane and C.-R. Sheue for excellent technical support and assistance on transverse sections and TEM processes; F.-C. Hsu for technical support on measurement of endogenous ABA levels; and C.-J. Chang and W.-M. Leu for their careful reading of the manuscript. This work was supported by the National Science Council (NSC 100-2321-B005-007-MY3) and Ministry of Education (ATU plan to J.-Y. Yang), Taiwan. Y.-P. Ho generated all of the transgenic plants and carried out electron microscopy experiments. C. M. Tan generated the X. campestris pv. campestris mutant strain and performed ABA experiments. M.-Y. Li generated antibodies and performed qRT-PCR and ABA analyses. H. Lin carried out confocal laser-scanning microscopy experiments. W.-L. Ding interpreted the results and supervised the project. J.-Y. Yang designed the experiments, analyzed the data, interpreted the results, and wrote the manuscript. All authors commented on the manuscript.

\section{LITERATURE CITED}

Aarts, N., Metz, M., Holub, E., Staskawicz, B. J., Daniels, M. J., and Parker, J. E. 1998. Different requirements for EDS1 and NDR1 by disease resistance genes define at least two $R$ gene-mediated signaling pathways in Arabidopsis. Proc. Natl. Acad. Sci. U.S.A. 95:10306-10311.

Adams-Phillips, L., Briggs, A. G., and Bent, A. F. 2010. Disruption of poly(ADP-ribosyl)ation mechanisms alters responses of Arabidopsis to biotic stress. Plant Physiol. 152:267-280.

Anders, S., and Huber, W. 2010. Differential expression analysis for sequence count data. Genome Biol. 11:R106.

Asselbergh, B., De Vleesschauwer, D., and Höfte, M. 2008. Global switches and fine-tuning-ABA modulates plant pathogen defense. Mol. 
Plant-Microbe Interact. 21:709-719.

Bari, R., and Jones, J. D. G. 2009. Role of plant hormones in plant defence responses. Plant Mol. Biol. 69:473-488.

Bartsch, M., Gobbato, E., Bednarek, P., Debey, S., Schultze, J. L., Bautor J., and Parker, J. E. 2006. Salicylic acid-independent ENHANCED DISEASE SUSCEPTIBILITY1 signaling in Arabidopsis immunity and cell death is regulated by the monooxygenase FMO1 and the nudix hydrolase NUDT7. Plant Cell 18:1038-1051.

Büttner, D., and Bonas, U. 2010. Regulation and secretion of Xanthomonas virulence factors. FEMS (Fed. Eur. Microbiol. Soc.) Microbiol. Rev. 34:107-133.

Canonne, J., Marino, D., Jauneau, A., Pouzet, C., Brière, C., Roby, D., and Rivas, S. 2011. The Xanthomonas type III effector XopD targets the Arabidopsis transcription factor MYB30 to suppress plant defense. Plant Cell 23:3498-3511.

Carviel, J. L., Al-Daoud, F., Neumann, M., Mohammad, A., Provart, N. J., Moeder, W., Yoshioka, K., and Cameron, R. K. 2009. Forward and reverse genetics to identify genes involved in the age-related resistance response in Arabidopsis thaliana. Mol. Plant Pathol. 10:621-634.

Castañeda, A., Reddy, J. D., El-Yacoubi, B., and Gabriel, D. W. 2005. Mutagenesis of all eight avr genes in Xanthomonas campestris pv. campestris had no detected effect on pathogenicity, but one avr gene affected race specificity. Mol. Plant-Microbe Interact. 18:1306-1317.

Chiasson, D., Ekengren, S., Martin, G., Dobney, S., and Snedden, W. 2005. Calmodulin-like proteins from Arabidopsis and Tomato are involved in host defense against Pseudomonas syringae pv. tomato. Plant Mol. Biol. 58:887-897.

Choi, D. S., and Hwang, B. K. 2011. Proteomics and functional analyses of pepper abscisic acid-responsive $1(A B R I)$, which is involved in cell death and defense signaling. Plant Cell 23:823-842.

Clough, S. J., and Bent, A. F. 1998. Floral dip: A simplified method for Agrobacterium-mediated transformation of Arabidopsis thaliana. Plant J. 16:735-743

Coll, N. S., Epple, P., and Dangl, J. L. 2011. Programmed cell death in the plant immune system. Cell Death Differ. 18:1247-1256.

Cui, J., Bahrami, A. K., Pringle, E. G., Hernandez-Guzman, G., Bender, C. L., Pierce, N. E., and Ausubel, F. M. 2005 Pseudomonas syringae manipulates systemic plant defenses against pathogens and herbivores. Proc. Natl. Acad. Sci. U.S.A. 102:1791-1796.

de Torres-Zabala, M., Truman, W., Bennett, M. H., Lafforgue, G., Mansfield, J. W., Rodriguez Egea, P., Bögre, L., and Grant, M. 2007. Pseudomonas syringae pv. tomato hijacks the Arabidopsis abscisic acid signalling pathway to cause disease. EMBO (Eur. Mol. Biol. Organ.) J. 26:1434-1443.

DeYoung, B. J., and Innes, R. W. 2006. Plant NBS-LRR proteins in pathogen sensing and host defense. Nat. Immunol. 7:1243-1249.

Dodds, P. N., and Rathjen, J. P. 2010. Plant immunity: Towards an integrated view of plant-pathogen interactions. Nat. Rev. Genet. 11:539-548.

Dunoyer, P., Himber, C., and Voinnet, O. 2006. Induction, suppression and requirement of RNA silencing pathways in virulent Agrobacterium tumefaciens infections. Nat. Genet. 38:258-263.

Ercetin, M. E., and Gillaspy, G. E. 2004. Molecular characterization of an Arabidopsis gene encoding a phospholipid-specific inositol polyphosphate 5-phosphatase. Plant Physiol. 135:938-946.

Fan, J., Hill, L., Crooks, C., Doerner, P., and Lamb, C. 2009. Abscisic acid has a key role in modulating diverse plant-pathogen interactions. Plant Physiol. 150:1750-1761.

Feys, B. J., Moisan, L. J., Newman, M. A., and Parker, J. E. 2001. Direct interaction between the Arabidopsis disease resistance signaling proteins, EDS1 and PAD4. EMBO (Eur. Mol. Biol. Organ.) J. 20:54005411

Frey, A., Effroy, D., Lefebvre, V., Seo, M., Perreau, F., Berger, A., Sechet, J., To, A., North, H. M., and Marion-Poll, A. 2012. Epoxycarotenoid cleavage by NCED5 fine-tunes ABA accumulation and affects seed dormancy and drought tolerance with other NCED family members. Plant J. 70:501-512

Głowacki, S., Macioszek, V., and Kononowicz, A. 2011. R proteins as fundamentals of plant innate immunity. Cell Mol. Biol. Lett. 16:1-24.

González-Guzmán, M., Apostolova, N., Bellés, J. M., Barrero, J. M., Piqueras, P., Ponce, M. R., Micol, J. L., Serrano, R., and Rodríguez, P. L. 2002. The short-chain alcohol dehydrogenase ABA2 catalyzes the conversion of xanthoxin to abscisic aldehyde. Plant Cell 14:1833-1846.

Gopalan, S., Bauer, D. W., Alfano, J. R., Loniello, A. O., and He, S. Y. Collmer, A. 1996. Expression of the Pseudomonas syringae avirulence protein AvrB in plant cells alleviates its dependence on the hypersensitive response and pathogenicity (hrp) secretion system in eliciting genotype-specific hypersensitive cell death. Plant Cell 8:1095-1105.

Gutierrez, L., Mauriat, M., Guénin, S., Pelloux, J., Lefebvre, J. F., Louvet, R., Rusterucci, C., Moritz, T., Guerineau, F., Bellini, C., and Van Wuytswinkel, O. 2008. The lack of a systematic validation of reference genes: A serious pitfall undervalued in reverse transcription-polymerase chain reaction (RT-PCR) analysis in plants. Plant Biotechnol. J. 6:609618.

Hatsugai, N., Iwasaki, S., Tamura, K., Kondo, M., Fuji, K., Ogasawara, K. Nishimura, M., and Hara-Nishimura, I. 2009. A novel membrane fusion-mediated plant immunity against bacterial pathogens. Genes Dev. 23:2496-2506.

Hsu, F. C., Chou, M. Y., Peng, H. P., Chou, S. J., and Shih, M. C. 2011. Insights into hypoxic systemic responses based on analyses of transcrip tional regulation in Arabidopsis. PLoS One 6:e28888. Published online.

Hwang, I., and Goodman, H. M. 1995. An Arabidopsis thaliana root-specific kinase homolog is induced by dehydration, $\mathrm{ABA}$, and $\mathrm{NaCl}$. Plant J. 8:37-43.

Hwang, S. G., Lin, N. C., Hsiao, Y. Y., Kuo, C. H., Chang, P. F., Deng, W. L., Chiang, M. H., Shen, H. L., Chen, C. Y., and Cheng, W. H. 2011. The Arabidopsis short-chain dehydrogenase/reductase 3, an ABSCISIC ACID DEFICIENT 2 homolog, is involved in plant defense responses but not in ABA biosynthesis. Plant Physiol. Biochem. 51:63-73.

Ishikawa, K., Yoshimura, K., Harada, K., Fukusaki, E., Ogawa, T., Tamoi, M., and Shigeoka, S. 2010. AtNUDX6, an ADP-ribose/NADH pyrophosphohydrolase in Arabidopsis, positively regulates NPR1-dependent salicylic acid signaling. Plant Physiol. 152:2000-2012.

Jensen, M. K., Kjaersgaard, T., Nielsen, M. M., Galberg, P., Petersen, K., O'Shea, C., and Skriver, K. 2010 The Arabidopsis thaliana NAC transcription factor family: Structure-function relationships and determinants of ANAC019 stress signalling. Biochem. J. 426:183-196.

Jiang, B. L., He, Y. Q., Cen, W. J., Wei, H. Y., Jiang, G. F., Jiang, W., Hang, X. H., Feng, J. X., Lu, G. T., Tang, D. J., and Tang, J. L. 2008. The type III secretion effector XopXccN of Xanthomonas campestris pv. campestris is required for full virulence. Res. Microbiol. 159:216220.

Jiang, W., Jiang, B. L., Xu, R. Q., Huang, J. D., Wei, H. Y., Jiang, G. F., Cen, W. J., Liu, J., Ge, Y. Y., Li, G. H., Su, L. L., Hang, X. H., Tang, D. J., Lu, G. T., Feng, J. X., He, Y. Q., and Tang, J. L. 2009. Identification of six type III effector genes with the PIP box in Xanthomonas campestris pv. campestris and five of them contribute individually to full pathogenicity. Mol. Plant-Microbe Interact. 22:1401-1411.

Kang, J., Hwang, J. U., Lee, M., Kim, Y. Y., Assmann, S. M., Martinoia, E., and Lee, Y. 2010. PDR-type ABC transporter mediates cellular uptake of the phytohormone abscisic acid. Proc. Natl. Acad. Sci. U.S.A. 107:2355-2360.

Kinch, L. N., Yarbrough, M. L., Orth, K., and Grishin, N. V. 2009. Fido, a novel AMPylation domain common to Fic, Doc, and AvrB. PLoS One 4:e5818. Published online.

Laloi, C., Mestres-Ortega, D., Marco, Y., Meyer, Y., and Reichheld, J. P. 2004. The Arabidopsis cytosolic thioredoxin $h 5$ gene induction by oxidative stress and its W-box-mediated response to pathogen elicitor. Plant Physiol. 134:1006-1016.

Lee, J. Y., Wang, X., Cui, W., Sager, R., Modla, S., Czymmek, K., Zybaliov, B., van Wijk, K., Zhang, C., Lu, H., and Lakshmanan, V. 2011. A plasmodesmata-localized protein mediates crosstalk between cell-to-cell communication and innate immunity in Arabidopsis. Plant Cell 23:3353-3373.

Li, J., Brader, G., and Palva, E. T. 2008. Kunitz trypsin inhibitor: An antagonist of cell death triggered by phytopathogens and fumonisin B1 in Arabidopsis. Mol. Plant 1:482-495.

Li, Y., Pennington, B. O., and Hua, J. 2009. Multiple $R$-like genes are negatively regulated by BON1 and BON3 in Arabidopsis. Mol. PlantMicrobe Interact. 22:840-848.

Lu, H. 2009. Dissection of salicylic acid-mediated defense signaling networks. Plant Signal. Behav. 4:713-717.

Meyers, B. C., Kozik, A., Griego, A., Kuang, H., and Michelmore, R. W. 2003. Genome-wide analysis of NBS-LRR-encoding genes in Arabidopsis. Plant Cell 15:809-834.

Mishina, T. E., and Zeier, J. 2006. The Arabidopsis flavin-dependent monooxygenase FMO1 is an essential component of biologically induced systemic acquired resistance. Plant Physiol. 141:1666-1675.

Mizuno, N., Hosogi, N., Park, P., and Takumi, S. 2010. Hypersensitive response-like reaction is associated with hybrid necrosis in interspecific crosses between tetraploid wheat and Aegilops tauschii Coss. PLoS One 5:e11326. Published online.

Munkvold, K. R., and Martin, G. B. 2009. Advances in experimental methods for the elucidation of Pseudomonas syringae effector function with a focus on AvrPtoB. Mol. Plant Pathol. 10:777-793.

Navarro, L., Dunoyer, P., Jay, F., Arnold, B., Dharmasiri, N., Estelle, M. Voinnet, O., and Jones, J. D. G. 2006. A plant miRNA contributes to antibacterial resistance by repressing auxin signaling. Science 312:436439.

Nawrath, C., Heck, S., Parinthawong, N., and Métraux, J. P. 2002. EDS5, an essential component of salicylic acid-dependent signaling for disease 
resistance in Arabidopsis, is a member of the MATE transporter family. Plant Cell 14:275-286.

Pieterse, C. M. J., Leon-Reyes, A., Van der Ent, S., and Van Wees, S. C. M. 2009. Networking by small-molecule hormones in plant immunity. Nat. Chem. Biol. 5:308-316.

Ren, X., Chen, Z., Liu, Y., Zhang, H., Zhang, M., Liu, Q., Hong, X., Zhu, J. K., and Gong, Z. 2010. ABO3, a WRKY transcription factor, mediates plant responses to abscisic acid and drought tolerance in Arabidopsis. Plant J. 63:417-429.

Reuber, T. L., and Ausubel, F. M. 1996. Isolation of Arabidopsis genes that differentiate between resistance responses mediated by the RPS 2 and RPM1 disease resistance genes. Plant Cell 8:241-249.

Robert-Seilaniantz, A., Navarro, L., Bari, R., and Jones, J. D. G. 2007. Pathological hormone imbalances. Curr. Opin. Plant Biol. 10:372-379.

Rong, W., Feng, F., Zhou, J., and He, C. 2010. Effector-triggered innate immunity contributes Arabidopsis resistance to Xanthomonas campestris. Mol. Plant Pathol. 11:783-793.

Salomon, D., Dar, D., Sreeramulu, S., and Sessa, G. 2011. Expression of Xanthomonas campestris pv. vesicatoria type III effectors in yeast affects cell growth and viability. Mol. Plant-Microbe Interact. 24:305-314.

Schulze-Lefert, P., and Robatzek, S. 2006. Plant pathogens trick guard cells into opening the gates. Cell 126:831-834.

Shang, Y., Li, X., Cui, H., He, P., Thilmony, R., Chintamanani, S., ZwieslerVollick, J., Gopalan, S., Tang, X., and Zhou, J. M. 2006. RAR1, a central player in plant immunity, is targeted by Pseudomonas syringae effector AvrB. Proc. Natl. Acad. Sci. U.S.A. 103:19200-19205.

Simpson, R. B., and Johnson, L. J. 1990. Arabidopsis thaliana as a host for Xanthomonas campestris pv. campestris. Mol. Plant-Microbe Interact. 3:233-237.

Song, J. T., Lu, H., and Greenberg, J. T. 2004. Divergent roles in Arabidopsis thaliana development and defense of two homologous genes, ABERRANT GROWTH AND DEATH2 and AGD2-LIKE DEFENSE RESPONSE PROTEIN1, encoding novel aminotransferases. Plant Cell 16:353-366

Spoel, S. H., and Dong, X. 2008. Making sense of hormone crosstalk during plant immune responses. Cell Host Microbe 3:348-351.

Tanaka, H., Osakabe, Y., Katsura, S., Mizuno, S., Maruyama, K., Kusakabe, K., Mizoi, J., Shinozaki, K., and Yamaguchi-Shinozaki, K. 2012. Abiotic stress-inducible receptor-like kinases negatively control ABA signaling in Arabidopsis. Plant J. 70:599-613.

van Wees, S. 2008. Phenotypic analysis of Arabidopsis mutants: Trypan blue stain for fungi, oomycetes, and dead plant cells. Cold Spring Harbor Protocols. doi: 10.1101/pdb.prot4982. Published online.

Verhage, A., van Wees, S. C. M., and Pieterse, C. M. J. 2010. Plant immunity: It's the hormones talking, but what do they say? Plant Physiol. 154:536-540.

Vicente, J., Cascán, T., Vicedo, B., García-Agustín, P., Hamberg, M., and Castresana, C. 2012. Role of 9-lipoxygenase and $\alpha$-dioxygenase oxylipin pathways as modulators of local and systemic defense. Mol. Plant 5:914-928.

von Saint Paul, V., Zhang, W., Kanawati, B., Geist, B., Faus-Keßler, T., Schmitt-Kopplin, P., and Schäffner, A. R. 2011. The Arabidopsis glucosyltransferase UGT76B1 conjugates isoleucic acid and modulates plant defense and senescence. Plant Cell 23:4124-4145.

Wang, G., Ellendorff, U., Kemp, B., Mansfield, J. W., Forsyth, A., Mitchell, K., Bastas, K., Liu, C. M., Woods-Tör, A., Zipfel, C., de Wit, P. J. G. M., Jones, J. D. G., Tör, M., and Thomma, B. P. H. J. 2008. A genomewide functional investigation into the roles of receptor-like proteins in
Arabidopsis. Plant Physiol. 147:503-517.

Wang, G. F., Seabolt, S., Hamdoun, S., Ng, G., Park, J., and Lu, H. 2011a. Multiple roles of WIN3 in regulating disease resistance, cell death, and flowering time in Arabidopsis. Plant Physiol. 156:1508-1519.

Wang, L., Tang, X., and He, C. 2007. The bifunctional effector AvrXccC of Xanthomonas campestris pv. campestris requires plasma membraneanchoring for host recognition. Mol. Plant Pathol. 8:491-501.

Wang, L., Tsuda, K., Truman, W., Sato, M., Nguyen, L. V., Katagiri, F. and Glazebrook, J. 2011b. CBP60g and SARD1 play partially redundant critical roles in salicylic acid signaling. Plant J. 67:1029-1041.

Wang, Y. H., Gehring, C., and Irving, H. R. 2011c. Plant natriuretic peptides are apoplastic and paracrine stress response molecules. Plant Cell Physiol. 52:837-850.

Weigel, D., and Glazebrook, J. 2006. Transformation of Agrobacterium using the freeze-thaw method. Cold Spring Harbor Protocols. doi: 10.1101/pdb.prot4666. Published online.

White, F. F., Potnis, N., Jones, J. B., and Koebnik, R. 2009. The type III effectors of Xanthomonas. Mol. Plant Pathol. 10:749-766.

Wildermuth, M. C., Dewdney, J., Wu, G., and Ausubel, F. M. 2001. Isochorismate synthase is required to synthesize salicylic acid for plant defence. Nature 414:562-565.

Williams, P. H. 1980. Black rot: A continuing threat to world crucifers Plant Dis. 64:736-742.

Wilton, M., and Desveaux, D. 2010. Lessons learned from type III effector transgenic plants. Plant Signal. Behav. 5:746-748.

Wright, H., van Doorn, W. G., and Gunawardena, A. H. 2009. In vivo study of developmental programmed cell death using the lace plant (Aponogeton madagascariensis; Aponogetonaceae) leaf model system. Am. J. Bot. 96:865-876.

Xin, Z., Wang, A., Yang, G., Gao, P., and Zheng, Z. L. 2009. The Arabidopsis A4 subfamily of lectin receptor kinases negatively regulates abscisic acid response in seed germination. Plant Physiol. 149:434-444.

Xu, R. Q., Blanvillain, S., Feng, J. X., Jiang, B. L., Li, X. Z., Wei, H. Y. Kroj, T., Lauber, E., Roby, D., Chen, B., He, Y. Q., Lu, G. T., Tang, D. J., Vasse, J., Arlat, M., and Tang, J. L. 2008. AvrACXcc8004, a type III effector with a leucine-rich repeat domain from Xanthomonas campestris pathovar campestris confers avirulence in vascular tissues of Arabidopsis thaliana ecotype Col-0. J. Bacteriol. 190:343-355.

Yang, H., Li, Y., and Hua, J. 2006a. The C2 domain protein BAP1 negatively regulates defense responses in Arabidopsis. Plant J. 48:238-248.

Yang, H., Yang, S., Li, Y., and Hua, J. 2007. The Arabidopsis BAP1 and $B A P 2$ genes are general inhibitors of programmed cell death. Plant Physiol. 145:135-146.

Yang, S., Yang, H., Grisafi, P., Sanchatjate, S., Fink, G. R., Sun, Q., and Hua, J. 2006b. The BON/CPN gene family represses cell death and promotes cell growth in Arabidopsis. Plant J. 45:166-179.

Yoo, S. D., Cho, Y. H., and Sheen, J. 2007. Arabidopsis mesophyll protoplasts: A versatile cell system for transient gene expression analysis. Nat. Protocols 2:1565-1572.

Zhang, K., Xia, X., Zhang, Y., and Gan, S. S. 2012. An ABA-regulated and Golgi-localized protein phosphatase controls water loss during leaf senescence in Arabidopsis. Plant J. 69:667-678.

Zhou, N., Tootle, T. L., Tsui, F., Klessig, D. F., and Glazebrook, J. 1998. PAD4 functions upstream from salicylic acid to control defense responses in Arabidopsis. Plant Cell 10:1021-1030.

Zuo, J., Niu, Q. W., and Chua, N. H. 2000. An estrogen receptor-based transactivator XVE mediates highly inducible gene expression in transgenic plants. Plant J. 24:265-273 